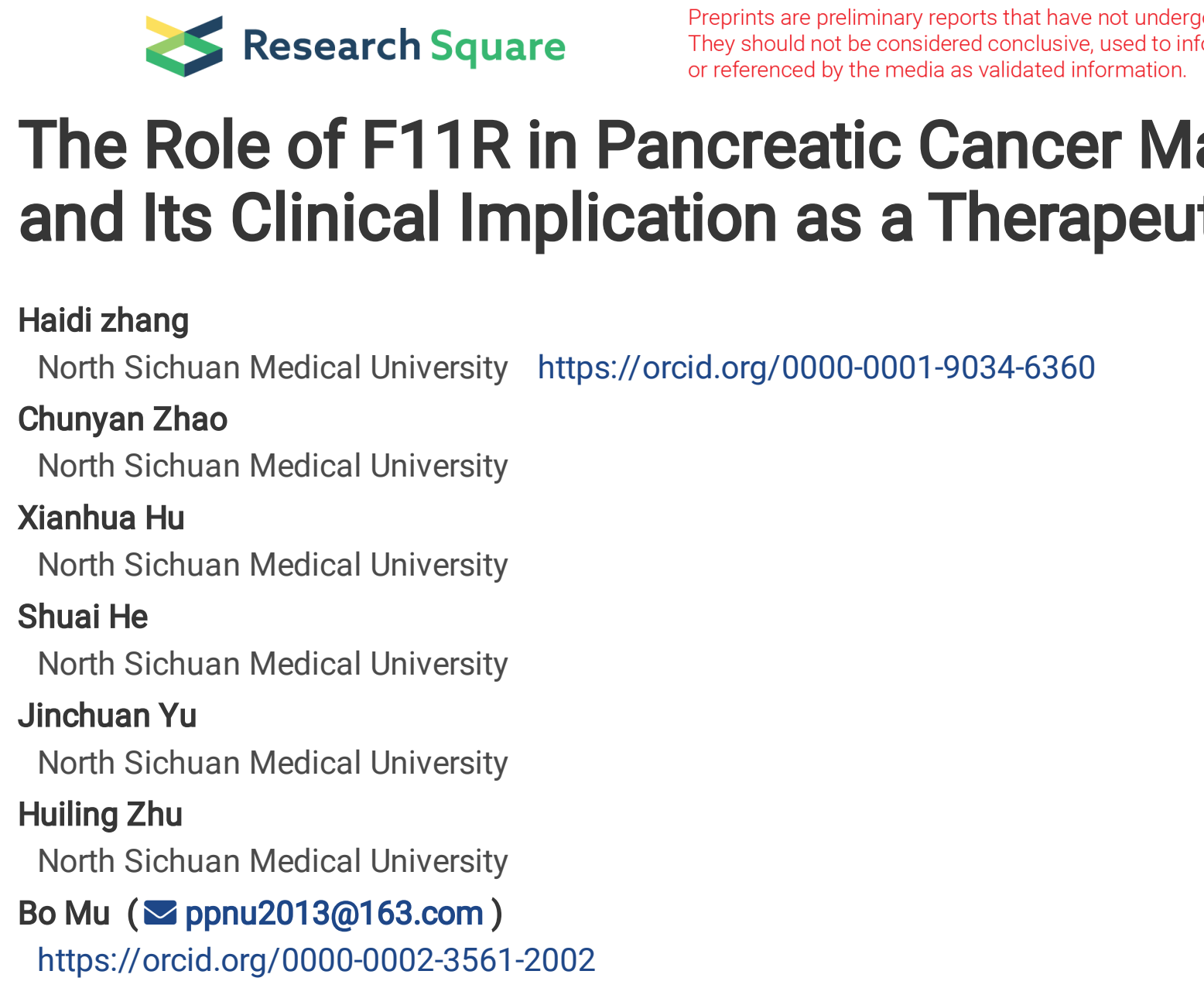

\section{Haidi zhang}

North Sichuan Medical University https://orcid.org/0000-0001-9034-6360

\section{Chunyan Zhao}

North Sichuan Medical University

Xianhua Hu

North Sichuan Medical University

Shuai He

North Sichuan Medical University

Jinchuan Yu

North Sichuan Medical University

Huiling Zhu

North Sichuan Medical University

Bo Mu (Dppnu2013@163.com )

https://orcid.org/0000-0002-3561-2002

\title{
The Role of F11R in Pancreatic Cancer Malignancy and Its Clinical Implication as a Therapeutic Target
}

\section{Research article}

Keywords: F11R (-/-); pancreatic cancer; genome editing; malignancy degree; cellular behavior

Posted Date: August 30th, 2019

DOI: https://doi.org/10.21203/rs.2.13324/v1

License: (c) (1) This work is licensed under a Creative Commons Attribution 4.0 International License.

Read Full License 


\title{
The Role of F11R in Pancreatic Cancer Malignancy and Its Clinical Implication
} as a Therapeutic Target

\author{
HaiDi Zhang ${ }^{1 \#}$ ChunYan Zhao ${ }^{2 \#}$ XianHua $\mathrm{Hu}^{3}$ Shuai $\mathrm{He}^{4}$ Jinchuan $\mathrm{Yu}^{4}$ \\ Huiling $\mathrm{Zhu}^{5}$ Bo Mu* \\ \# represent co-first author \\ * represent corresponding author
}

\begin{abstract}
Background

The F11 receptor belongs to the immunoglobulin superfamily and is expressed in epithelial and endothelial cells. F11R mediates the formation of tight junctions between the epithelium and endothelium, and participates in the invasion and metastasis of tumor cells. We have previously shown that the F11R gene is closely related to KRas $(\mathrm{P}=0.76)$, a known therapeutic target for pancreatic cancer $(\mathrm{PCa})$. In recent years, it has been found that F11R is expressed in different tumors and has biological effects.However, according to different tumor cases, different cell lines and experimental conditions, the regulatory results and mechanisms of F11R on tumor are different, even contradictory,and the expression, clinical significance and biological mechanism of F11R in tumor tissues have not been reported in detail.
\end{abstract}

Results

To investigate the role of F11R in carcinogenesis of PCa and the potential of F11R as a therapies target for PCa, we silenced F11R (-/-) in the PCa cell line PANC-1 (known to express high levels of KRas) using lentiviral approaches.We found that F11R silencing led to decreased cell proliferation, a loss of cell invasiveness, reduced colony forming ability, cell cycle arrest in G1 phase, cells apoptosis enhanced, and ros enhanced. In vitro data showed that inhibition of F11R decreased proliferation and invasiveness of cancer cells. The present results suggest that F11R may be a promising therapeutic target for PCa. 


\section{Conclusions}

This study used bioinformatics combined with gene chip data to find the gene F11R, which is closely related to KRAS gene, and we used lentivirus to package shRNA plasmid to interfere with the gene F11R in pancreatic cancer panc-1 cells. A series of biobehavioral studies indicated the biobehavioral function and malignancy of panc-1 in pancreatic cancer cells with negative regulation of F11R gene.Based on this, we need to continue to clarify the expression of F11R gene in clinical case samples to determine whether F11R gene can be a new therapeutic target for pancreatic cancer.

Keywords : F11R (-/-);pancreatic cancer;genome editing;malignancy degree;cellular behavior

\section{Background}

Pancreatic cancer $(\mathrm{PCa})$ is one of the most aggressive malignant tumors that remains difficult to diagnose and treat. Although progress has been made regarding PCa epidemiology, the mortality rates of PCa patients remain high, and the 5-year survival rates are low (1). The early clinical symptoms of PCa are not obvious, and early detection is rare. Surgical resection with adjuvant chemotherapy remains the mainstay of curative treatment,due to the high degree of malignancy and rapid progression of $\mathrm{PCa}$, the optimal treatment time has often passed upon diagnosis (2). Early diagnostic and more effective intervention strategies are required to improve the survival rates of PCa patients.

Malignant tumor formation is both multi-stage and progressive. Tumorigenesis is driven by endogenous mutations to genes and exogenous cancer-promoting factors. Many tumor cells gradually evolve into clinically visible pathological forms. The activation of proto-oncogenes coupled to the inactivation of tumor suppressor genes are known drivers of tumorigenesis (3). Proto-oncogenes are a class of potentially carcinogenic genes that regulate cell growth, differentiation, and apoptosis. Proto-oncogenes have low levels of expression in normal cells, but when overexpressed promote cancer formationis (4). Like other malignant tumors, $\mathrm{PCa}$ is 
caused by external stimuli that disrupts the balance between proto-oncogenes and tumor suppressor genes.

Human F11R receptor belongs to the immunoglobulin superfamily and promotes tight junction formation between epithelial and endothelial cells is (5). F11R is a 27 KD protein that promotes tumor metastasis and embryogenesis, widely expressed in neutrophils, monocytes, platelets and lymphocytes is (6). F11R regulates epithelial and endothelial cell movement, leukocyte migration, platelet activation, and cell barrier integrity is (7). The regulation of F11R is closely related to its ability to dimerize mediated through intracellular PDZ binding motif is (8).

In the present study, we quantitatively measured the expression of F11R in five pancreatic cancer cell lines(MIA paca-2, bxpc-3, cfpac-1, SW1990, PANC-1) by real-timePCR. F11R level in tumor specimens from PCa was increased significantly compared with level in nonneoplastic tissues.To investigate the role of F11R in carcinogenesis, immunohistochemical studies were done in pancreatic intraepithelial neoplasias.In addition, to evaluate the functional role of F11R and its possible therapeutic implications,we inhibited expression of F11R using RNA interference(RNAi) and investigated its effect on proliferation and invasiveness, at the same time, its effect on cell cycle and apoptosis of pancreatic cancer cells in vitro.

F11R is expressed in a range of tumors but its role in tumorigenesis remains controversialis (9-11). Histopathological and cytological studies on endometrial carcinoma, prostate cancer, renal cell carcinoma, and some breast cancer cases have shown that low F11R expression positively correlates with tumor cell invasion and motility, tumor grade, stage, and poor prognosis is (12).In contrast, histopathological, cytological, and microRNA analysis suggested that F11R expression in breast and endometrial carcinoma are negatively correlated is (13). The clinical significance and biological roles of F11R in tumor tissue has not been explored in detail. In this study, we silenced F11R in the PCa cell line PANC-1 and assessed its oncogenic potential.Taken together, the data suggest that F11R is a promising therapeutic targets for PCa. 


\section{Methods}

The PCa cell line PANC-1,MIA paca-2, bxpc-3, cfpac-1, SW1990 were purchased from the cell bank of the Chinese Academy of Sciences in Shanghai.Tissue samples were obtained from 30 patients who underwent surgery of the pancreas between July, 2013 and July, 2018 at the first affiliated hospital of North Sichuan Medical College,Nanchong, China. Written informed consent was obtained from all patients, and the study was conducted according to the Helsinki Declaration.Fetal Bovine Serum (FBS) was purchased from HyClone, USA. High glucose DMEM was purchased from Gibco, USA. Trypsin-EDTA (0.25\%) was purchased from Gibco. Mouse anti-human F11R monoclonal antibodies and rabbit anti-human GAPDH monoclonal antibodies were purchased from Santa Cruz Corporation. HRP-labeled goat anti-rabbit antibodies and goat anti-mouse antibodies were purchased from Beijing Zhongshan Jinqiao Biotechnology Co., Ltd. Penicillin, streptomycin and ampicillin were purchased from Shanghai Shenggong engineering Co., Ltd.

\section{Bioinformatics analysis genechips of PCa cells}

Affymetrix expression profiling gene chip of $\mathrm{PCa}$ cells were purchase by Shanghai Genechem Co., LTD(Shanghai China).In this study, gene chips of PCa cells were produced to screen genes of importance to PCa development and KRas signaling. F11R was found to be highly expressed in all PCa samples.In addition to, using the TCGA database, we found that F11R expression varied according to stage. In addition, lower F11R expression was an indicator of prolonged survival.

\section{Immunohistochemical studies}

Thirty tumoral tissues were obtained from patients who underwent surgery for pancreatic cancer.Sections of formalin-fixed,paraffin-embedded specimens were deparaffinized in xylene anddehydrated in graded alcohol. Endogenous peroxidase activity wasblocked with 3\% hydrogen peroxide in PBS.Incubated with the appropriate dilution of mouse monoclonal F11R (Santa Cruz Corporation) antibody overnight at $4{ }^{\circ} \mathrm{C}$.Sections were then incubated with the appropriate dilution of 
biotinylated anti-mouse $\operatorname{IgG}$ (Vectastain Elite Avidin-Biotin Complex kit, Vector Labs,Burlingame, CA) for 30 minutes.Immunocomplexes were visualized with stable Thermo Scientific ${ }^{\mathrm{TM}}$ UltraVision ${ }^{\mathrm{TM}}$ Quanto HRP DAB (Thermo Fisher Scientific , China).The sections were rinsed with tap water and counterstained with hematoxylin for 12 seconds.

\section{RNAi lentiviruses transduction}

Cells were transfected with F11R RNAi lentiviruses synthesized by Shanghai GeneChem Co Ltd. Lentiviral vectors expressing green fluorescence protein (GFP) were used as controls. Silencing was confirmed through two primer pairs:

\begin{tabular}{|c|l|}
\hline (1) & $\begin{array}{l}\text { 5'-CCGGTTCTCCGAACGTGTCACGTTTCAAGAGAACGTGACACGTT } \\
\text { CGGAGAATTTTTG-3' }\end{array}$ \\
\hline$(2)$ & $\begin{array}{l}\text { GV112-NC-2:5'-AATTCAAAAATTCTCCGAACGTGTCACGTTCTCTT } \\
\text { GAAACGTGACACGTTCGGAGAA-3' }\end{array}$ \\
\hline $\begin{array}{l}\text { Scrambled } \\
\text { controls }\end{array}$ & 5'-TTCTCCGAACGTGTCACGT-3' \\
\hline
\end{tabular}

HEK293T cells were transfected with GV248 plasmids and viruses were harvested 72 hours post-transfection (hpi). Cells were infected with lentiviruses encoding F11R and $\mathrm{NC}$ at a multiplicity of infection (MOI) of 10, as per the manufacturer's instructions. HT-29 cell transductants were selected for 1 week using puromycin (Thermo Fisher Scientific, $2 \mu \mathrm{g} / \mathrm{mL})$.

\section{Western blot analysis}

Total proteins from infected cells were extracted and protein expression was determined by western blot analysis. Cell lysates containing 5 x SDS sample buffer were boiled at $95{ }^{\circ} \mathrm{C}$ for $5 \mathrm{~min}$ and $30 \mathrm{ug}$ protein was resolved by SDS PAGE. Proteins were wet-transferred to 2.5um PVDF membranes at $200 \mathrm{~mA}$ for $2 \mathrm{~h}$ and blocked in 5\% milk in PBS-T for 1 hour. Membranes were probed with primary antibodies overnight at $4{ }^{\circ} \mathrm{C}$ and labeled with the appropriate secondary antibodies at 
room temperature for $1 \mathrm{~h}$. Protein bands were visualized using the BeyoECL system and imaged on a fluorescence imager.

\section{Quantitative real-time PCR}

Total RNA was extracted from five pancreatic cancer cell lines using RNAiso Plus reagent (Takara). Reverse transcription was performed using the PrimeScript RT reagent kit (Takara). The resulting cDNA arrays (Shanghai GeneChem), were used to examine the mRNA levels of F11R or GAPDH using an ABI 7500 Fast Real-Time PCR System (Applied Biosystems) and the SYBR Premix Ex Tag (Takara). PCR conditions were as follows (two step method):Pretesttemperature of $95{ }^{\circ} \mathrm{C}$, for $15 \mathrm{~s}, 95^{\circ} \mathrm{C}$ for $5 \mathrm{~s}$, annealing and extension at $60{ }^{\circ} \mathrm{C}, 30 \mathrm{~s}$ (40 cycles). Light absorption values were measured during the stretch phase.

\section{Cell proliferation assays}

Cell proliferation was assessed using the Cell Counter Kit-8 (CCK-8 kit) by Shanghai Beyotime Biotechnology (Shanghai, China). Briefly, PANC-1 negative control RNAi-GFP (NC) and stable RNAi-F11R cells were plated at a density of $3 \times 10^{3}$ cells per well of a 96-well plate, and 10ul of CCK8 reagent was added to the cells for $2 \mathrm{~h}$. Absorbance's were measured at $450 \mathrm{~nm}$.

\section{Colony forming assays}

Cells that can divide and form clones are both adherent and proliferative. The clone formation rate reflects two important characters of the cell population, namely dependence and proliferation ability. NC and RNAi-F11R cells were plated into 600-well plates containing DMEM plus 10\% FBS. Colonies were visible after $\sim 1$ week. The media was removed, cells were washed in PBS, and fixed with 4\% paraformaldehyde for $20 \mathrm{~min}$. Colonies were stained in $0.1 \%$ crystal violet for $2 \mathrm{~h}$ and imaged using Carestream Molecular Imaging software. Colony formation rates were calculated as follows: $(\%)=$ number of clone's generated/the total number of inoculated cells x $100 \%$. 


\section{Flow cytometry}

Cells in the logarithmic phase were detached with trypsin and cell suspensions were treated with cell cycle and apoptosis analysis kits (C1052). Stained cells were analyzed on an ACEA NovoCyte.

\section{Apoptosis analysis}

After 72 hours of lentivirus transfection, cells in the logarithmic phase were detached with trypsin and cell suspensions were labeled with the Annexin v-PI apoptosis assay kit (C1065M Beyotime Shanghai). Samples were analyzed on an ACEA NovoCyte.

\section{Invasion Assays}

For transwell assays, CHEMICON Cell Invasion Assays were performed in an Invasion Chamber, consisting of a 24 -well tissue culture plate with 12 cell culture inserts. The inserts contain an $8 \mu \mathrm{m}$ pore size polycarbonate membrane, over which a thin layer of ECMatrixTM was dried. The ECM layer occludes the membrane pores, blocking non-invasive cells from migrating through the membrane. Invasive cells can however migrate through the ECM layer and cling to the bottom of the polycarbonate membrane. Cells $\left(1 \times 10^{4}\right)$ were added to the Transwells and FBS-containing medium (500 ul) was added to the lower chamber. Cells were cultured for 24 hours and the upper chamber was stained with $0.1 \%$ crystal violet. Migrating cells were imaged on a Leica DC 300F microscope, and count by Image J.

\section{Reactive oxygen species (ROS)}

Cells were seeded at a density of $1 \times 10^{4}$ cells in six-well plates and treated with DCFH-DA after 24 hrs. DCFH-DA is hydrolyzed by intracellular esterases into DCFH, which is oxidized to fluorescent DCF by ROS. The fluorescence intensity of DCF is therefore proportional to ROS production. Cells were treated with DCFH-DA in serum-free medium and cells were washed in PBS. The OD values were measured at 
$525 \mathrm{~nm}$.

\section{Statistical analysis}

Images were analyzed on Image $\mathbf{J}$ (National Institutes of Health).Statistical analysis were performed using SPSS 13.0. Data were compared using an unpaired two tailed Student's T-test. A $\mathrm{P}<0.05(*)$ and $\mathrm{P}<0.01(* *)$ were considered statistically significant.

\section{Results and discussions}

\section{Bioinformatics analysis of F11R about pancreatic cancer}

Upon bioinformatics analysis of F11R expression in 179 pancreatic cancer tissues and 171 normal tissues, the expression of F11R was significantly higher in pancreatic cancer (Fig 1A).Through bioinformatics analysis of the gene chip data, F11R was found to be highly expressed in PCa. GO enrichment analysis of the differentially expressed mRNAs revealed a high correlation between F11R and KRas $(\mathrm{Tab} 1, \mathrm{p}=0.76) . \mathrm{F} 11 \mathrm{R}$ expression in the pancreatic cancer tissue also varied according to stage and grade,the expression of F11R in pancreatic cancer patients of different ages were also assessed (Fig 1B).Data from the TCGA database showed that low F11R expression can prolong the overall survival rates of pancreatic cancer patients(Fig 1C).

\section{F11R expression in pancreatic cancer cell lines and PCa pathological specimens}

To confirm expression of F11R in pancreatic cancer cell lines, the expression of F11R in 5 pancreatic cancer cell lines was analyzed by q-PCR, and F11R was highly expressed(Fig 2A).For subsequent experiments, we selected PANC-1 cells because its expressed moderate levels of F11R. A total of 30 clinical pancreatic cancer paraffin pathological specimens from 2013 to 2018 were assessed, and 300 pathological sections were produced, 5 of which were collected for immunohistochemical staining. The expression of F11R in the samples reached $86 \%$ (Fig 2B). The results between the groups were consistent, demonstrating that F11R was highly expressed not only in 
pancreatic cancer cell lines, but also in the pathological tissues of pancreatic cancer patients.

Tabel 1 The gene highly correlated with KRas

\begin{tabular}{|c|c|}
\hline Name & $\mathrm{R}$ \\
\hline F11R & 0.76 \\
\hline CTTNBP2NL & 0.68 \\
\hline ADD3 & 0.67 \\
\hline HNRNPR & 0.65 \\
\hline CRIM1 & 0.62 \\
\hline MYNN & 0.61 \\
\hline ATP8B1 & 0.61 \\
\hline ABHD17C & 0.59 \\
\hline GRAMD3 & 0.59 \\
\hline MBOAT1 & 0.59 \\
\hline
\end{tabular}

Tab 1:Microarray analysis of PANC-1 cells showed that F11R was the gene most highly correlated with KRas $(P=0.76)$.

A

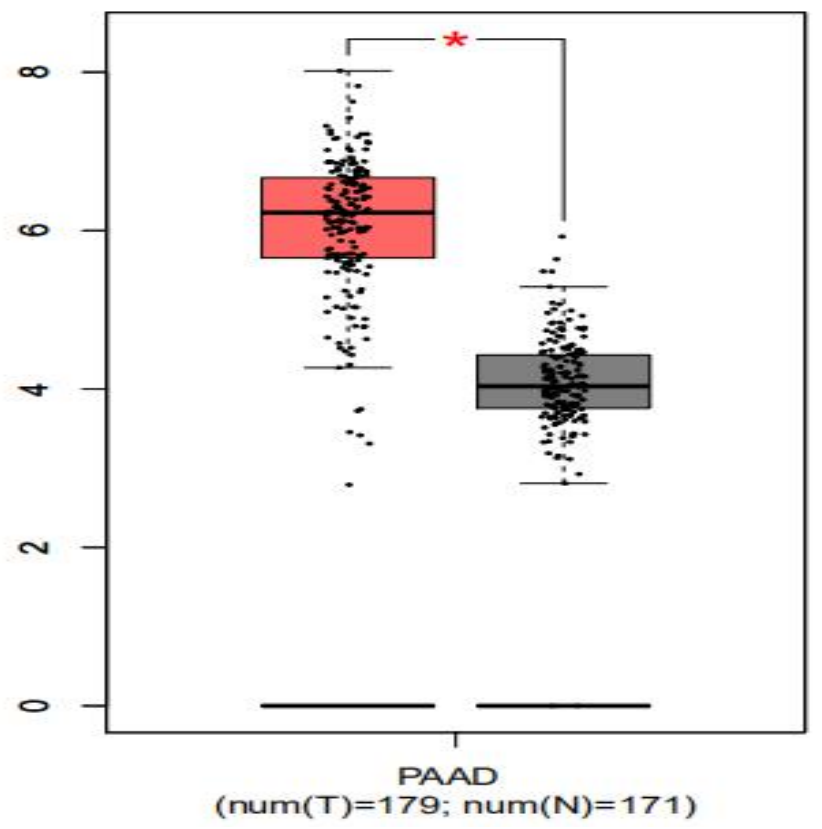


Cancer stages

Expression of $F 11 R$ in PAAD based on individual cancer stages

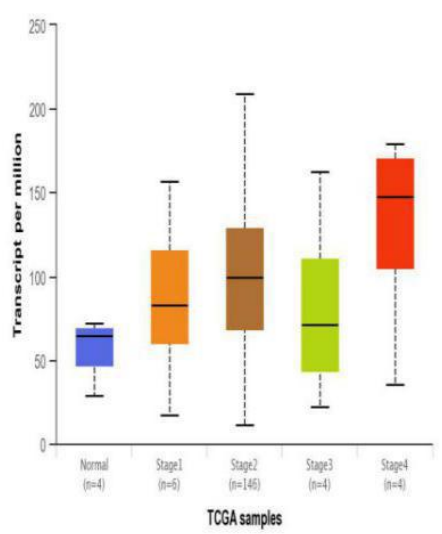

Tumor grade

Expression of F11R in PAAD based on tumor grade

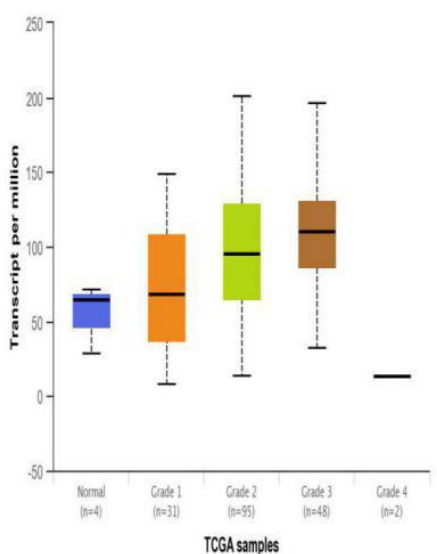

Patient's age

Expression of F11R in PAAD based on patient's age

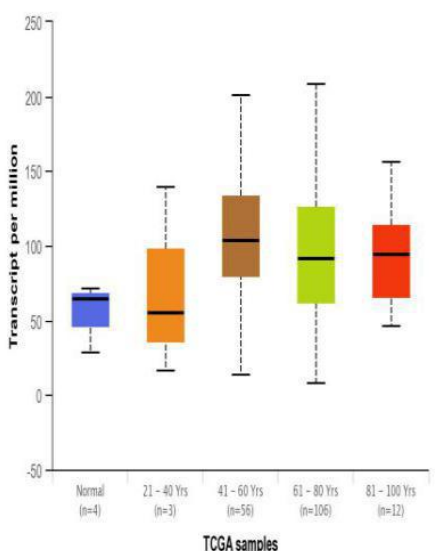

C

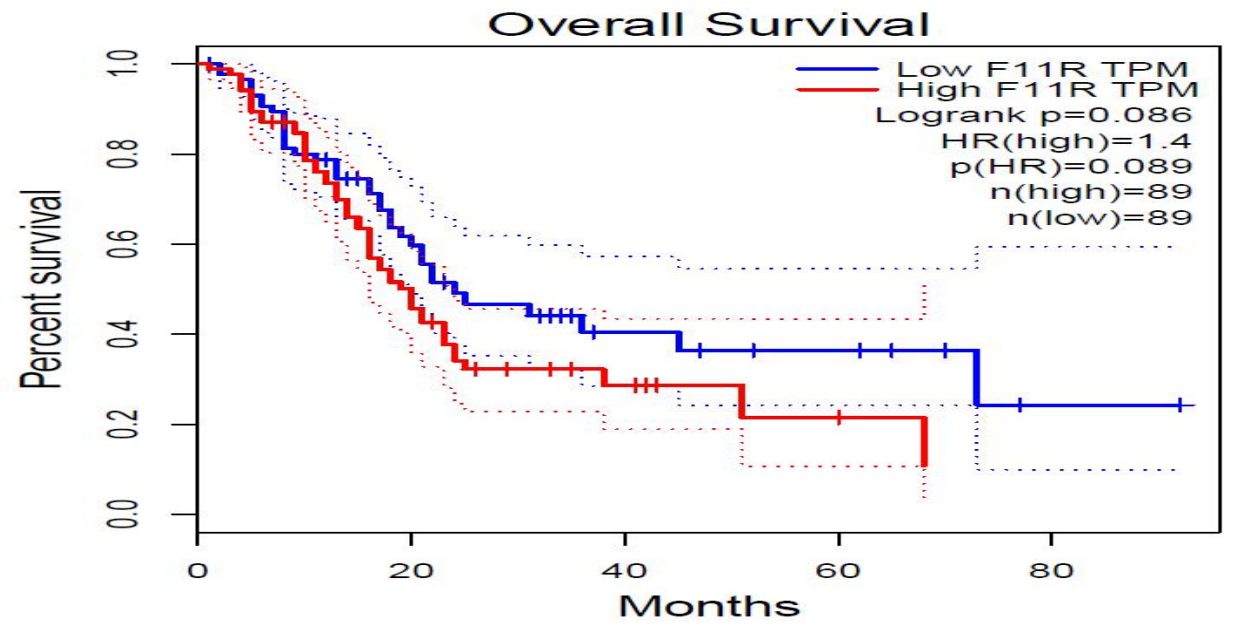

Figure 1. Bioinformatics analysis of F11R about pancreatic cancer. A: Bioinformatics analysis showed that the expression of F11R in pancreatic cancer was higher than that of normal tissue ( $P$ $<0.05)$. B: Data from the TCGA database showing the variable expression of F11R in pancreatic cancer tissue from different stages, different grades and different age. C:Data from the TCGA database showed that low expression of F11R prolongs the OS of pancreatic cancer patients. 


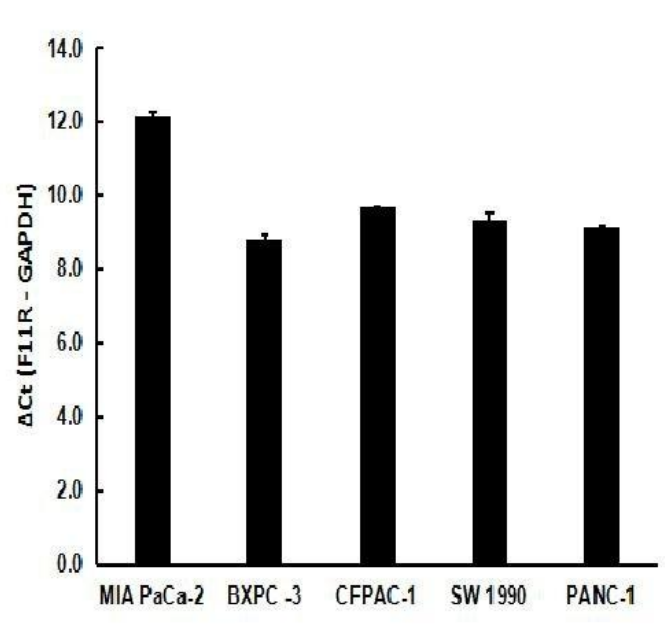

A

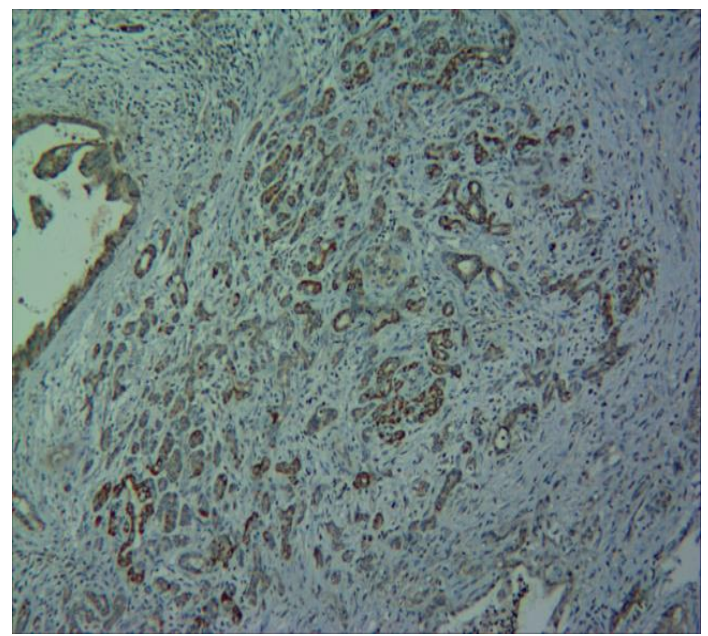

B

Figure 2. F11R expression in pancreatic cancer cell lines and PCa pathological

specimens.A:q-PCR showed that F11R is highly expressed in 5(PANC-1,MIA paca-2, bxpc-3,

cfpac-1, SW1990)pancreatic cancer cell lines.B: Immunohistochemical staining of F11R in

pathological specimens of pancreatic cancer showed that the expression of F11R was $86 \%$.

\section{Lentiviral transduction and Confirmation}

The transfection efficiency of the lentiviruses reached more than $90 \%$ in PANC-1 cells. Western blot analysis of F11R expression in PANC1 cells transfected with lentiviruses showed that the expression of F11R proteins were significantly lower after lentivirus transfection. 
A

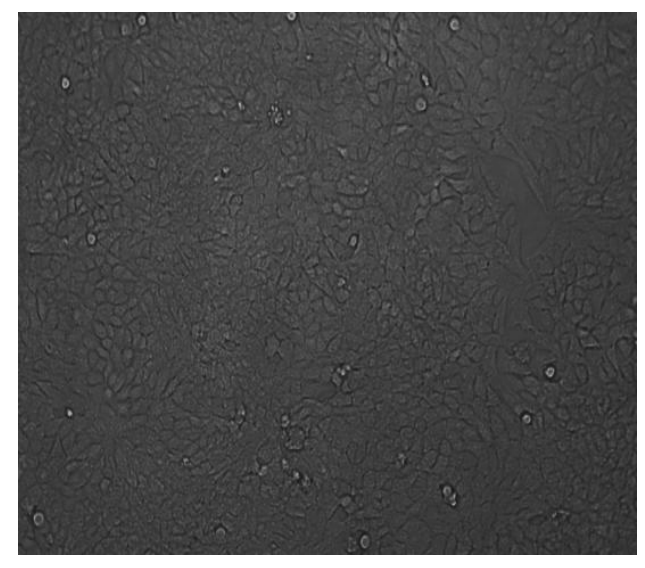

RNAi-F11R White

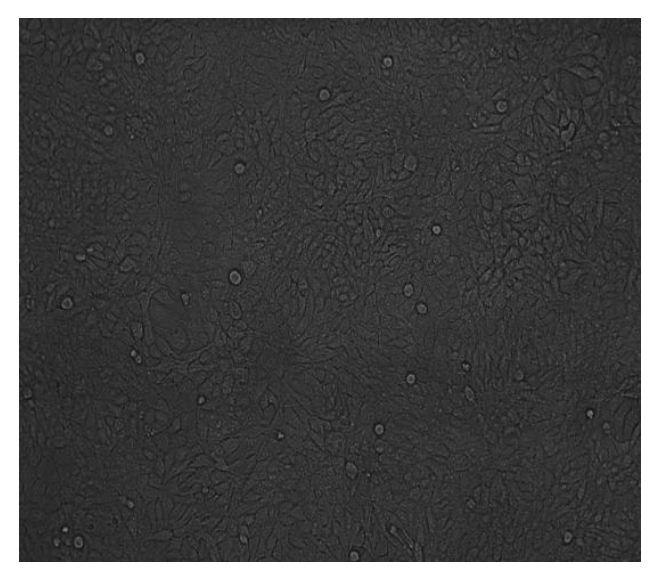

B

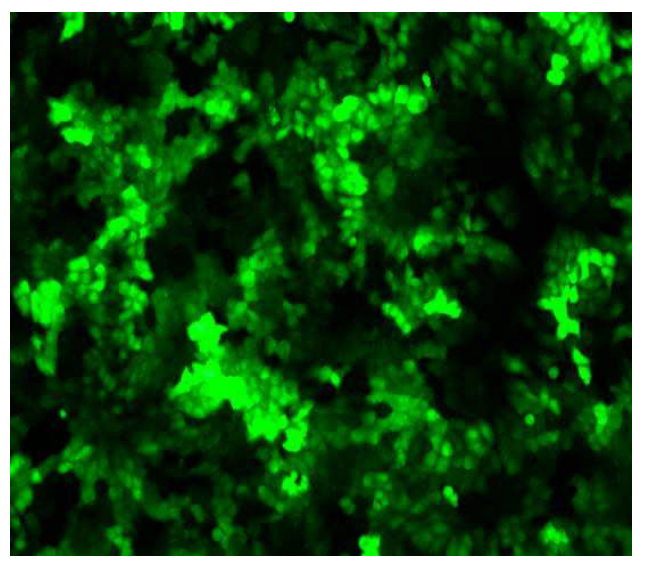

RNAi-F11R FLU

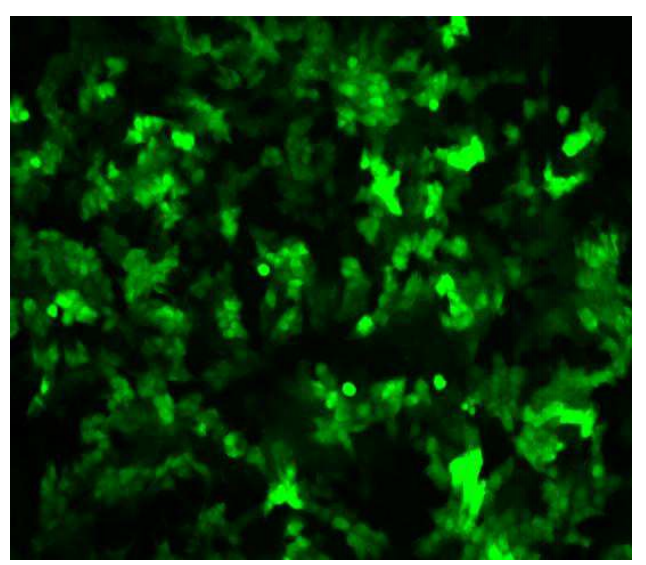

NC

RNAi-F11R

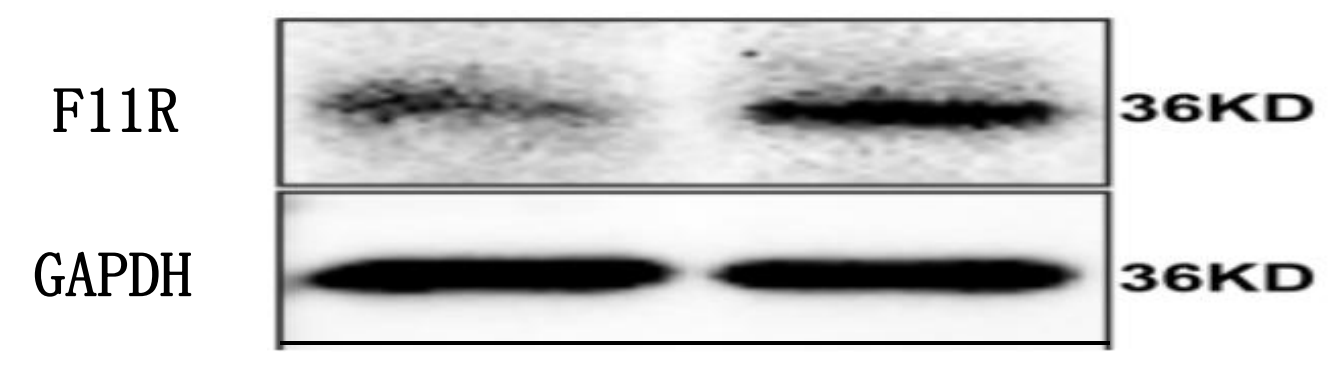


Figure 3. Knockout of PANC-1 F11R following lentivirus transfection.A: PANC-1 cells were transfected with control and F11R -/- lentiviruses for $72 \mathrm{~h}$. GFP fluorescence showed an infection efficiency $\geq 90 \%$. B: PANC-1 cells infected with the indicated lentiviruses for $72 \mathrm{~h}$ were assessed for F11R expression by western blot analysis. F11R expression in the F11R -/ -group was significantly reduced.

\section{Cell proliferation}

Enhanced cell proliferation is a characteristic feature of tumor cells. Cell Counting Kit-8 (Figure 4A) and colony formation assays (Figure 4B) were performed to assess the proliferation of PANC-1 F11R -/- cells. Comparable proliferation rates were observed between Control and NC cells. However, cell numbers in the F11R -/group were significantly lower than the control and NC groups, indicating reduced proliferation $(\mathrm{P}<0.05)$.

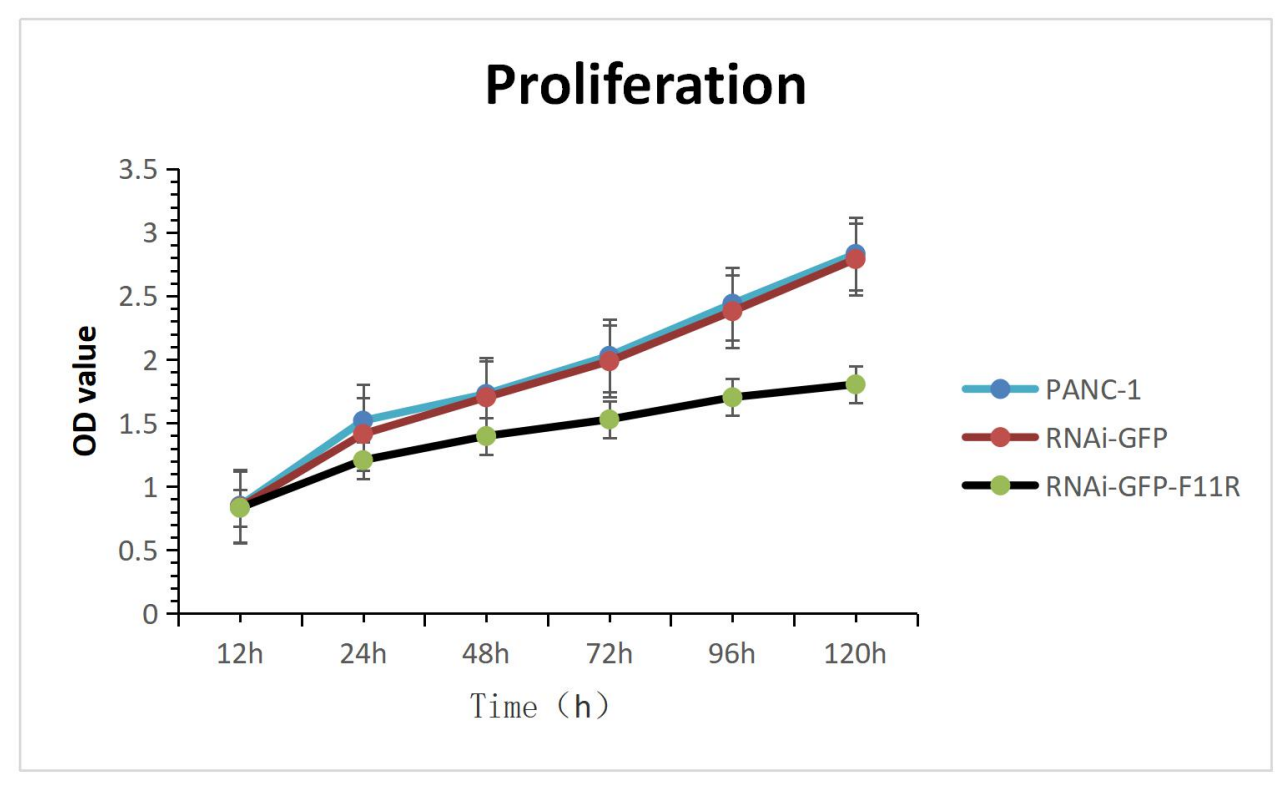



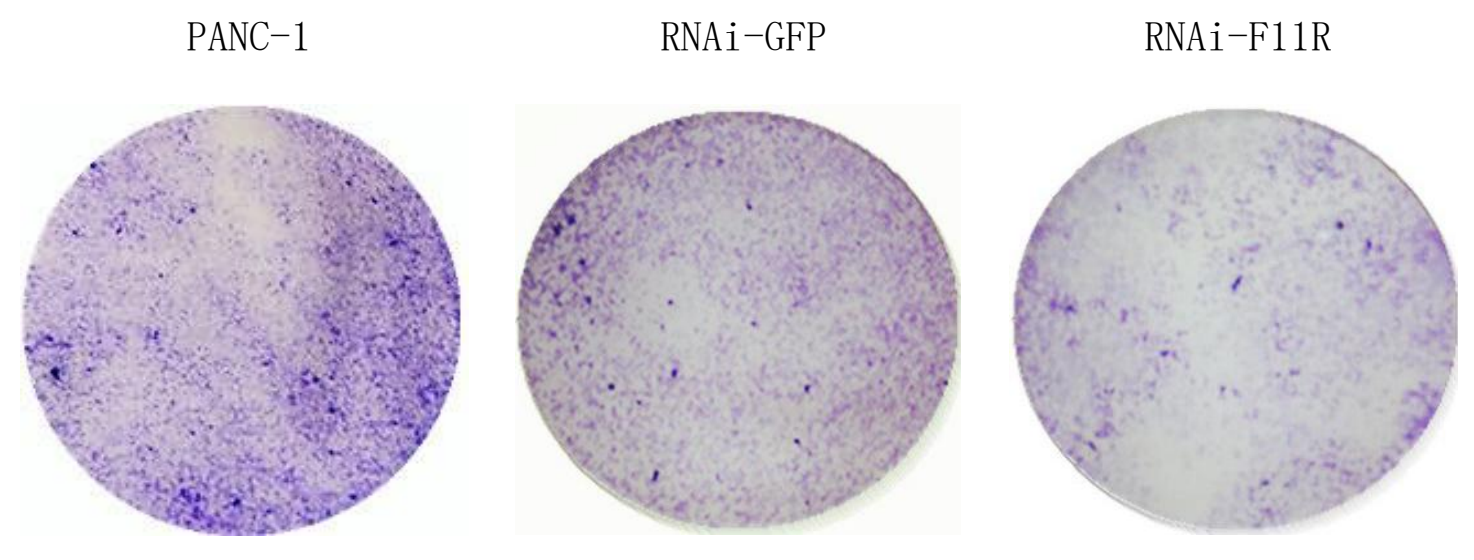

Colony

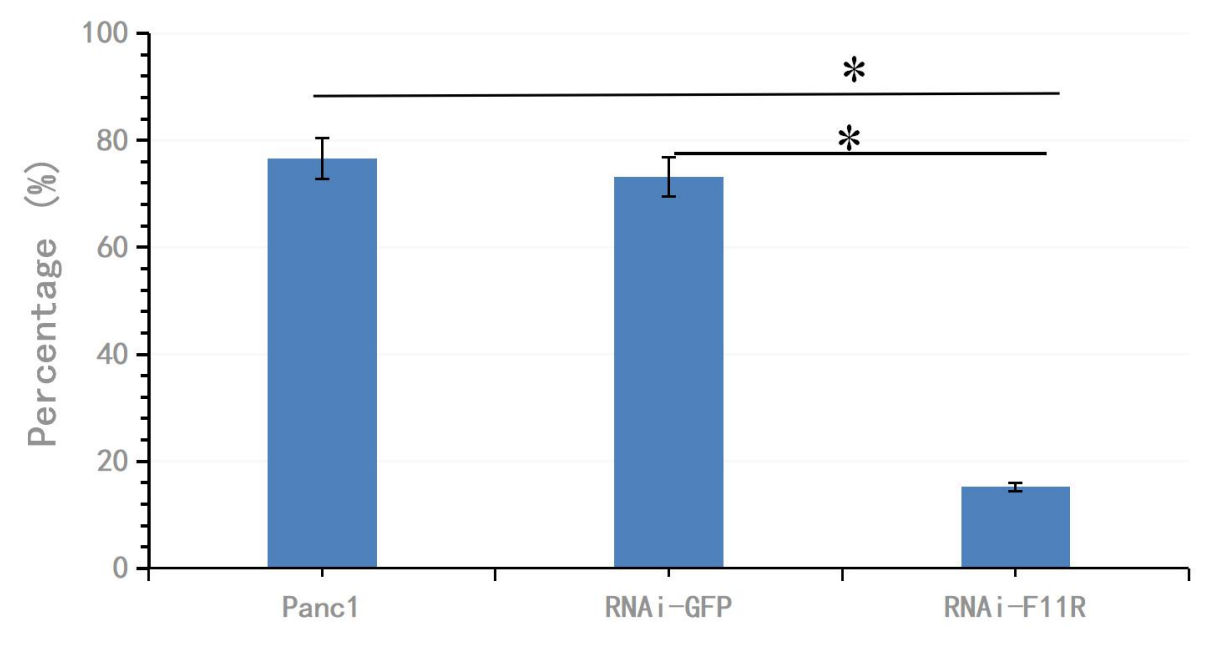

B

Figure 4. Cells proliferation assays. FigA:Cell proliferation in the F11R -/- group was significantly slower than the blank control and NC groups. FigB: The number of clones in the negative control group (NC) was $78.32 \%$ which decreased to $15.24 \%$ in the RNAi-GFP group. These results suggest that F11R silencing significantly inhibits the clonal formation of PANC-1 cells (* represent $\mathrm{P}<0.05)$.

\section{Flow cytometry}

Flow cytometry (FCM) was used to assess the cell cycle distribution of PANC-1 F11R -/- cells. Compared to control and NC groups, the F11R -/- group showed higher levels of GO/G1 arrest, and lower S-phase progression (Figure 5). The G2 phase remained unchanged by F11R silencing. 
PANC-1
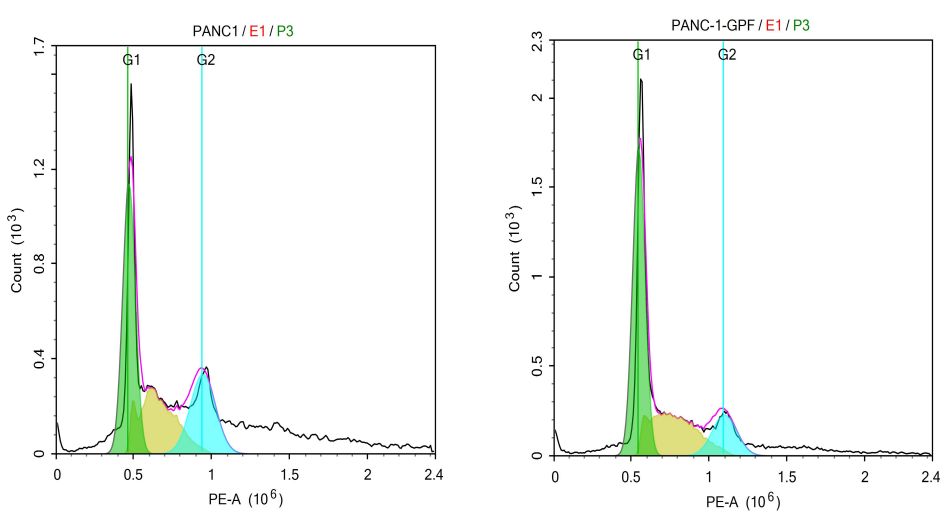

Cycle
RNAi-F11R

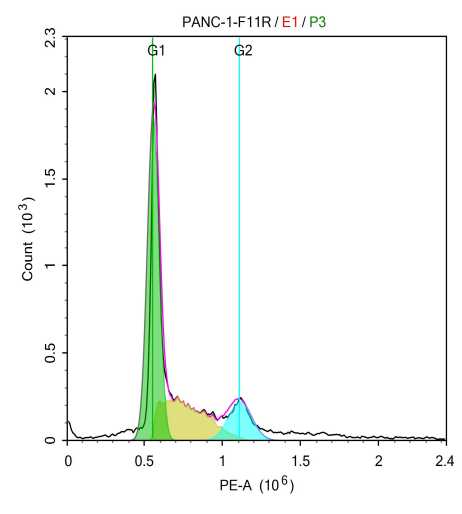

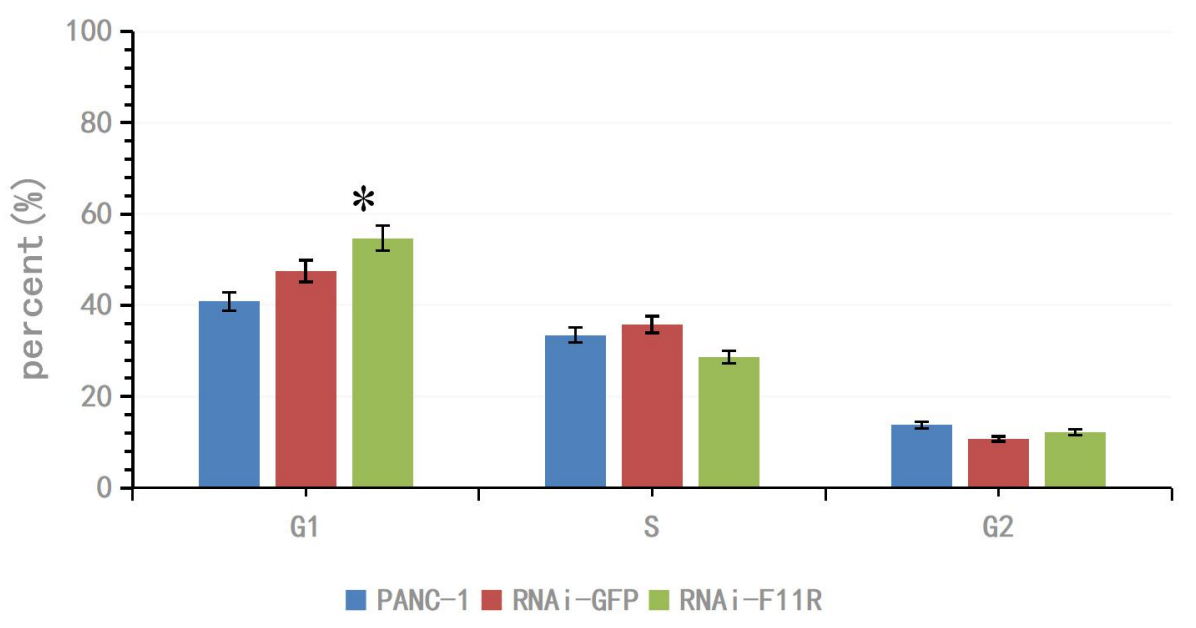

Figure 5. Flow cytometry analysis. Transfection increased the number of cells in the G0/G1 phase.

Cytotoxicity was significantly higher in F11R -/- cells compared to control or NC groups. F11R silencing led to further $\mathrm{G} 0 / \mathrm{G} 1$ phase arrest.(* represent $\mathrm{P}<0.05)$

Results as shown in the figure, the F11R -/- cells significantly blocked pancreatic cancer cell cycle at G0/G1 phase (panc-1 36.29 $\pm 4.11 \%$, RNAi-GFP $31.98 \pm 0.16 \%$, RNAi-F11R 50.56 $\pm 2.96 \%$ ), with statistically significant differences $(\mathrm{p}<0.05)$.At the same time, $\mathrm{G} 2$ cells were significantly reduced (panc-1 36.42 $\pm 2.29 \%$, RNAi-GFP $25.01 \pm 0.32 \%$, RNAi-F11R $15.08 \pm 0.6 \%$ ), while S cells had no significant effect (panc-1 20.47 $\pm 2.25 \%$, RNAi-GFP $35.85 \pm 0.38 \%$, RNAi-F11R $29.78 \pm 2.8 \%$ ), and there was no statistical difference ( $p>0.05$ ). 


\section{Apoptosis assays}

Cancer cells are typically refractory to apoptotic stimuli. Annexin v-PI apoptosis assay kits (C1065M Beyotime Shanghai) were used to assess apoptotic induction in control, NC, and F11R -/- cells by flow cytometry (Figure 6).

PANC-1

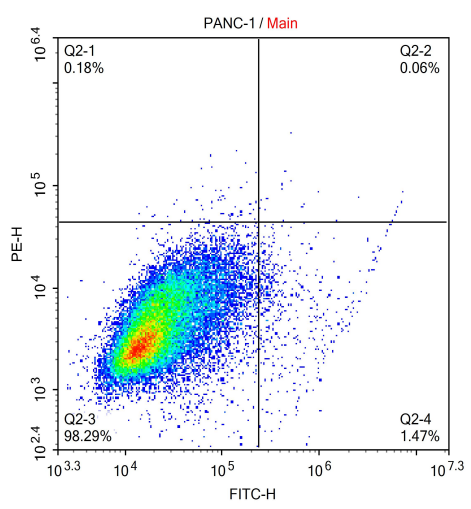

RNAi-GFP

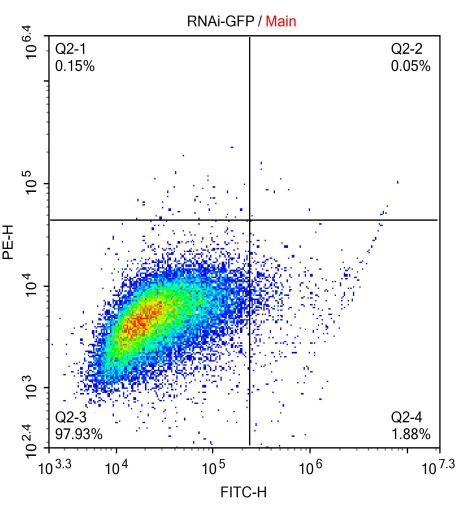

RNAi-F11R

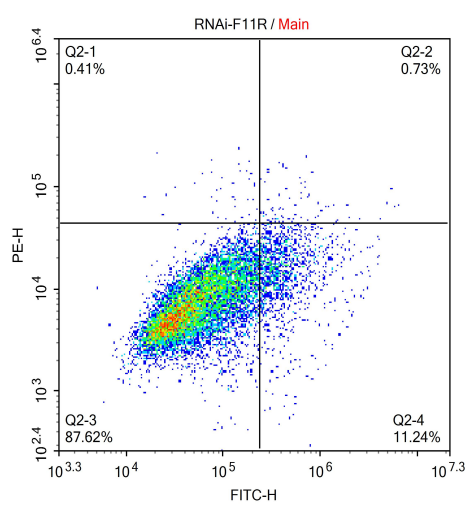

Apoptos is

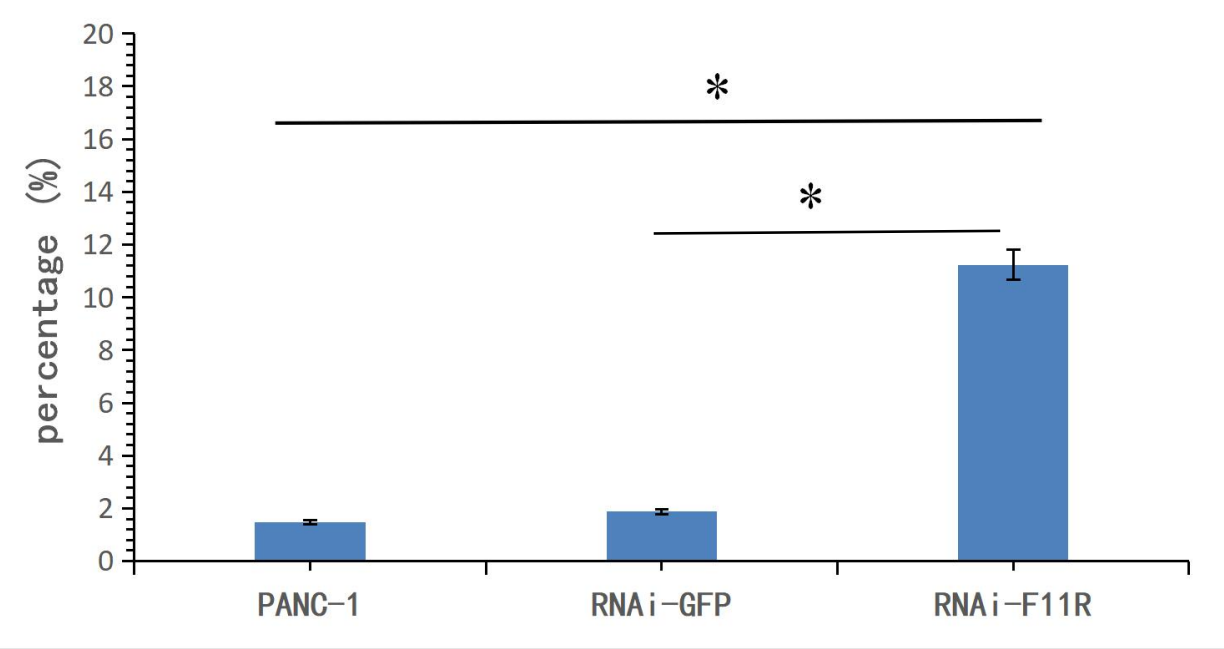

Figure 6. Apoptosis assessments. The number of apoptotic cells significantly increased following F11R silencing (lower right quadrant), as did the number of necrotic cells (upper right quadrant) .Results as shown in the figure, the proportion of apoptotic cells in the untreated group was $0.74 \%$, and the proportion of apoptotic cells in the early stage of RNAi-GFP was $1.86 \%$, showing no statistical difference compared with the untreated group.The proportion of RNAi-F11R apoptotic cells in F11R -/- cells was 15.85\%.Compared with untreated group and NC group, the proportion of dead cells in F11R -/- cells increased, with statistically significant 
differences. $(*$ represent $\mathrm{P}<0.05)$

\section{Invasion assays}

Transwell assays showed comparable numbers of control and NC cells penetrating the membrane (Figure 7). However, in the F11R -/- group, the number of cell migrating across the transmembrane significantly decreased. Thus F11R silencing in PANC-1 cells inhibits the invasion and migration ability of PANC-1 cells in vitro.

PANC-1

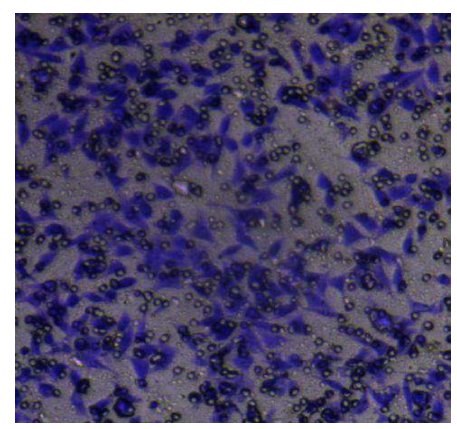

RNAi-GFP

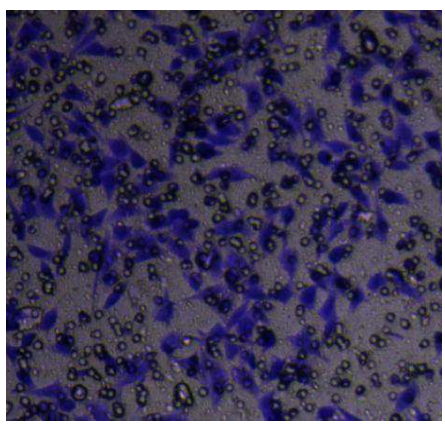

RNAi-F11R

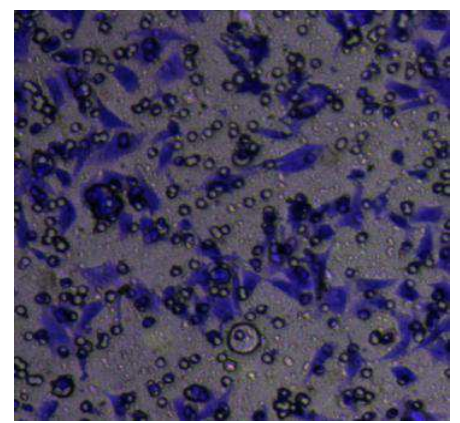

Invasion

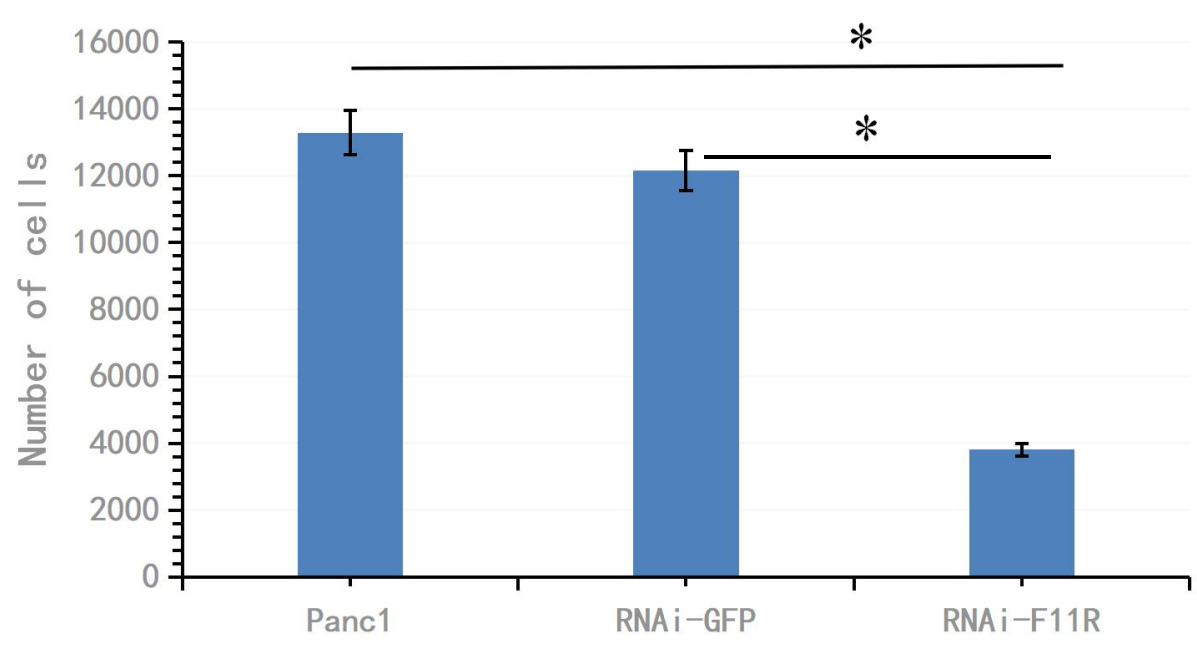

Figure 7. PANC-1 cell migration and invasion in vitro.In the F11R -/- group, the number of cells Transwell assay significantly decreased, indicating a loss of invasion. Cell numbers were calculated using ImageJ. Transwell assays showed that the number of migrating cells significantly decreased following F11R silencing. (* represent $\mathrm{P}<0.05)$ 


\section{Reactive oxygen species production in PANC-1 cells}

DCFH-DA was used to measure relative ROS production in control, NC, and F11R -/- cells (Figure 8). Increased fluorescent intensity (and thus elevated ROS production) were observed in F11R -/- cells $(\mathrm{P}<0.05)$ compared to control and NC cells.

PANC-1
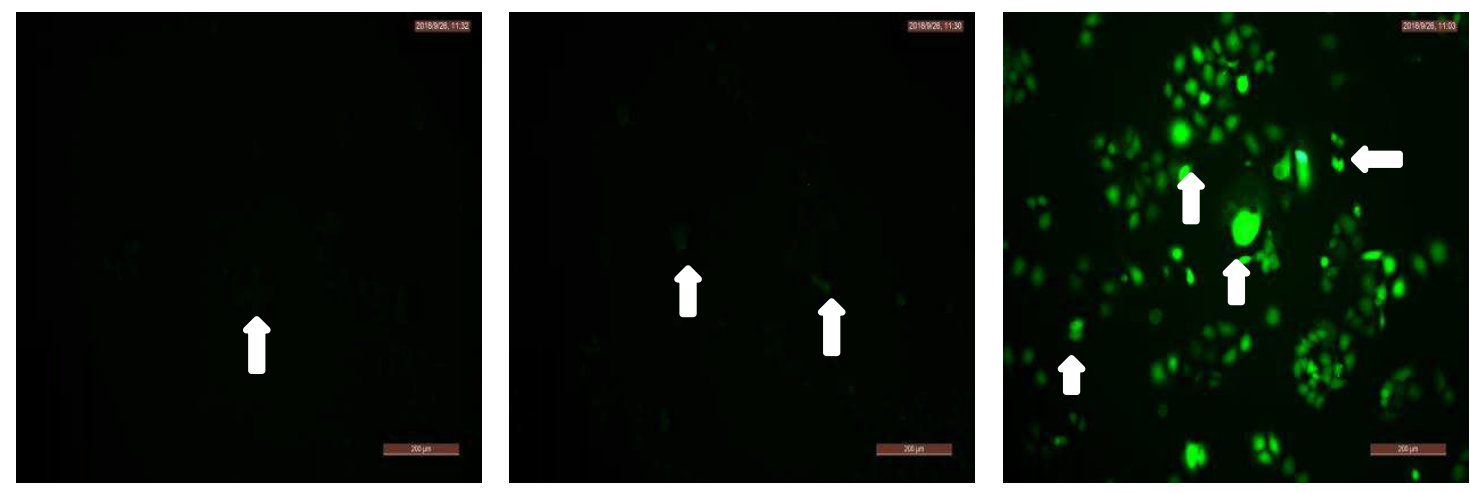

ROS

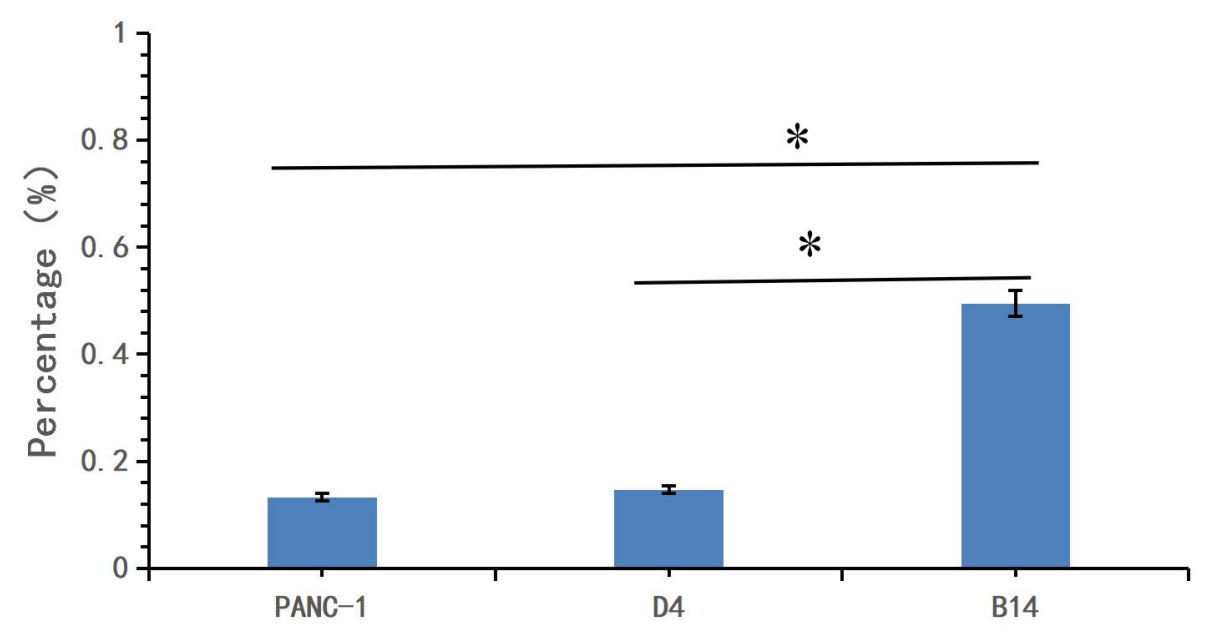

Figure 8. ROS production in PANC-1 cells. Fluorescence intensity represents intracellular ROS levels. ROS production was assessed through comparison of the fluorescent intensities of DCFH-DA staining in Control, NC and F11R groups. $(*$ represent $\mathrm{P}<0.05)$

\section{Conclusion}

This is a report of quantitative analyses of F11R levels in pancreatic cancer cells and pancreatic tissues from patients with PCa. F11R expression levels in PCa 
cells(PANC-1,MIA paca-2, bxpc-3, cfpac-1, SW1990) and carcinoma samples were significantly higher than those in nontumoral tissues.Bioinformatics-based quantitative analysis of gene chip revealed that F11R was differentially expressed in different stages, tumor grade and age of pancreatic cancer(14). At the same time,reducing the expression of F11R can prolong the survival rate of pancreatic cancer patients(15).These phenomena are very interesting,worth exploring further.In this study, we focused on the F11R gene as an anti-PCa target due to the ineffectiveness of current KRas therapies(16). We silenced F11R (-/-) in the PCa cell line PANC-1 using lentiviral approaches. Cell proliferation assays, cell invasion assays, colony formation assays, and transwell assays were performed to observe the biological behavior PANC-1 F11R -/- cells. Flow cytometry was used to assess cell cycle status and apoptosis of PANC-1 F11R -/- cells. We found that F11R silencing led to decreased cell proliferation, a loss of cell invasiveness, reduced colony forming ability, cell cycle arrest in G1 phase, cells apoptosis enhanced, and ros enhanced. We suspect that F11R can be used as a therapeutic target for pancreatic cancer .

With pancreatic cancer mortality remaining unchanged over the past decades, there is a clear impetus to develop novel therapies for the treatment of $\mathrm{PCa}(17-20)$.The molecular mechanisms of $\mathrm{PCa}$ are complex. Mutations accumulate in tumor-related genes, including proto-oncogenes and tumor suppressors, leading to the malignant transformation of normal pancreatic epithelial cells to form intraepithelial neoplasia of pancreatic carcinoma (pancreatic intraepithelial neoplasia, PanIN)(21-23). For early PCa detection, surgery can remove the tumor and other pathological tissue, but the lack of early PCa symptoms means most patients are diagnosed during late disease stages, the postoperative survival rates of PCa are low (15\% to 25\%) (24-26). Recent advances in gene therapies offer novel opportunities for treatment, based on previous studies of PCa genes(27-31).KRas is implicated in PCa development and its reduced expression can reduce the degree of malignancy(32). The production of KRAS specific inhibitors is challenging, and many inhibitors lack specificity and affinity(33-35). In addition, the RAS protein lacks small molecules that can target 
natural binding sites is.After 30 years of in-depth research, no Ras inhibitors are clinically available (36). We previously investigated dysregulated genes in PCA using bioinformatics and gene chip data,and discovered that F11R, a gene closely related to KRAS $(p=0.76)$, was significantly upregulated in PCA tissue. This provides a new idea for this study -- to solve the problem of KRAS as a gene therapy target in the application of PCa by interfering with F11R.

The F11R receptor belongs to the immunoglobulin superfamily and is expressed in epithelial and endothelial cells(37-40). F11R mediates the formation of tight junctions between the epithelium and endothelium, and participates in the invasion and metastasis of tumor cells. F11R localizes to microtubules and plays an important role in cell to cell adhesion(41).Given its association with pancreatic cancer stage and grade, this holds utility for the early diagnostic assessments. Two stable cell lines (RNAi-GFP and RNAi-F11R) were constructed using lentiviral vectors in this report.F11R silencing inhibited the proliferation and colony forming ability of PANC-1 cells. Scratch assays and transwell chamber perforation assessments showed that F11R silencing decreased the migration ability of PANC-1 cells. F11R increased the number of PANC-1 cells in the G0/G1 phase, but the proportion of cells in the S phase decreased. Flow cytometry analysis also revealed that F11R silencing enhanced PANC-1 apoptosis.The culmination of these assays revealed the potential of F11R as a novel PCa therapeutic target.

Bioinformatics strategies were based on the interconnectivity of intracellular signaling networks, which may also be applicable to other malignant tumors(42-43). Compared to surgery, radiotherapy, and chemotherapy, gene therapy has many advantages, including low cytotoxicity, low side effects, and high tolerance. There are already reports that,F11R antagonists as a therapeutic agents for the prevention and treatment of thrombosis, atherosclerosis, heart attacks, stroke and other clinical disorders(44-45), but whether F11R antagonists can be used in treating malignant tumors is still unknown.The identification of F11R as a novel anti-PCa target therefore holds interest from the perspective of gene therapy and precision treatment. 
Goetsch found that F11R monoclonal antibodies could reduce MCF-7 breast cancer cell proliferation is (46). Phosphoinositide 3-kinase (PI3K) and protein kinase C (PKC) inhibitors F11R-induced migration providing mechanistic insight into its oncogenic effects (47). The effects of F11R antibodies in PCa have not been investigated. The behavioral changes in PCa cells caused by the low expression of F11R remain unclear, but may be related to the immunologic responses of F11R. This will be the focus of our future PCa studies.

\section{Additional files}

Additional file 1: Table 1.Microarray analysis of PANC-1 cells showed that F11R was the gene most highly correlated with KRas $(P=0.76)$.

Additional file 2:Figure 1. Bioinformatics analysis of F11R about pancreatic cancer. A: Bioinformatics analysis showed that the expression of F11R in pancreatic cancer was higher than that of normal tissue $(P<0.05)$. B: Data from the TCGA database showing the variable expression of F11R in pancreatic cancer tissue from different stages, different grades and different age. C:Data from the TCGA database showed that low expression of F11R prolongs the OS of pancreatic cancer patients.

Additional file 3: Figure 2. F11R expression in pancreatic cancer cell lines and PCa pathological specimens.A:q-PCR showed that F11R is highly expressed in 5(PANC-1,MIA paca-2, bxpc-3, cfpac-1, SW1990)pancreatic cancer cell lines.B: Immunohistochemical staining of F11R in pathological specimens of pancreatic cancer showed that the expression of F11R was $86 \%$.

Additional file 4 : Figure 3. Knockout of PANC-1 F11R following lentivirus transfection.A: PANC-1 cells were transfected with control and F11R -/- lentiviruses for $72 \mathrm{~h}$. GFP fluorescence showed an infection efficiency $\geq 90 \%$. B: PANC-1 cells infected with the indicated lentiviruses for $72 \mathrm{~h}$ were assessed for F11R expression by western blot analysis. F11R expression in the F11R -/ -group was significantly reduced.

Additional file 5: Figure 4. Cells proliferation assays. FigA:Cell proliferation in the F11R -/group was significantly slower than the blank control and NC groups. FigB: The number of clones in the negative control group (NC) was $78.32 \%$ which decreased to $15.24 \%$ in the RNAi-GFP 
group. These results suggest that F11R silencing significantly inhibits the clonal formation of PANC-1 cells $(\mathrm{P}<0.05)$.

Additional file 6: Figure 5. Flow cytometry analysis. Transfection increased the number of cells in the G0/G1 phase. Cytotoxicity was significantly higher in F11R -/- cells compared to control or NC groups. F11R silencing led to further G0/G1 phase arrest.

Results as shown in the figure, the F11R -/- cells significantly blocked pancreatic cancer cell cycle at G0/G1 phase (panc-1 36.29 $\pm 4.11 \%$, RNAi-GFP $31.98 \pm 0.16 \%$, RNAi-F11R 50.56 $\pm 2.96 \%$ ), with statistically significant differences $(\mathrm{p}<0.05)$.At the same time, G2 cells were significantly reduced (panc-1 36.42 $\pm 2.29 \%$, RNAi-GFP $25.01 \pm 0.32 \%$, RNAi-F11R $15.08 \pm 0.6 \%$ ), while S cells had no significant effect (panc-1 20.47 $\pm 2.25 \%$, RNAi-GFP $35.85 \pm 0.38 \%$, RNAi-F11R $29.78 \pm 2.8 \%$ ), and there was no statistical difference $(\mathrm{p}>0.05)$.

Additional file 7: Figure 6. Apoptosis assessments. The number of apoptotic cells significantly increased following F11R silencing (lower right quadrant ), as did the number of necrotic cells (upper right quadrant) .Results as shown in the figure, the proportion of apoptotic cells in the untreated group was $0.74 \%$, and the proportion of apoptotic cells in the early stage of RNAi-GFP was $1.86 \%$, showing no statistical difference compared with the untreated group.The proportion of RNAi-F11R apoptotic cells in F11R -/- cells was 15.85\%.Compared with untreated group and NC group, the proportion of dead cells in F11R -/- cells increased $(\mathrm{P}<0.05)$, with statistically significant differences.

Additional file 8: Figure 7. PANC-1 cell migration and invasion in vitro.In the F11R -/- group, the number of cells Transwell assay significantly decreased, indicating a loss of invasion. Cell numbers were calculated using ImageJ. Transwell assays showed that the number of migrating cells significantly decreased following F11R silencing $(\mathrm{P}<0.05)$.

Additional file 9: Figure 8. ROS production in PANC-1 cells. Fluorescence intensity represents intracellular ROS levels. ROS production was assessed through comparison of the fluorescent intensities of DCFH-DA staining in Control, NC and F11R groups.

\section{Abbreviations}


PCa:pancreatic cancer

F11R:F11 receptor

PI3K:Phosphoinositide 3-kinase

PKC:protein kinase C

KRas:kirsten rat sarcoma viral oncogene

PBS:Phosphate buffered saline

RNAi:RNAinterference

DMEM; Dulbecco's modified eagle medium

DMSO: Dimethyl sulfoxide

\section{Acknowledgements}

We acknowledge the funding provided to those research by the Nanchong applied technology research and development fund project (16YFZJ0125);Nanchong school-school cooperative scientific research special project (NSMC20170402;18SXHZ0400) north sichuan medical research and development program (cby15-a-yb08). We also acknowledge the Pathological sections provided by the pathology department of north sichuan medical college. The funding boards had no role in thestudy design, data collection and analysis, decision to publish or preparationof the manuscript.

\section{Funding}

Nanchong applied technology research and development fund project (16YFZJ0125);Nanchong school-school cooperative scientific research special project (NSMC20170402;18SXHZ0400) north sichuan medical research and development program (cby15-a-yb08).

\section{Availability of data and materials}

All the data presented in this study is provided free and open to be used,included in the Supplementary Files that are quoted and described alongthe manuscript. 
Authors' contributions

Data curation, Haidi Zhang and Bo Mu; Funding acquisition, ChunYan Zhao and Bo Mu; Investigation, XianHua Hu; Methodology, Haidi Zhang, Shuai He, JinChuan Yu and Huiling Zhu; Software, Haidi Zhang; Writing - original draft, Haidi Zhang; Writing - review \& editing, Bo Mu.

\section{Ethics approval and consent to participate}

Ethics approval and consent to participate is "not applicable", because this work does not include samples from new patients or donors. All the information and data of human pathological section used in this work come from datasets already public in open repositories.

\section{Consent for publication}

Not applicable.

\section{Competing interests}

Not applicable.

\section{Publisher's Note}

Springer Nature remains neutral with regard to jurisdictional claims inpublished maps and institutional affiliations.

\section{Author details}

HaiDi Zhang\#:North sichuan medical college, no. 234 fujiang road, shunqing district, nanchong city,sichuan province, China.Institution:Basic medical college of north sichuan medical college.Email:1561858364@qq.com

ChunYan Zhao: North sichuan medical college, no. 234 fujiang road, shunqing district, nanchong city,sichuan province, China.Institution:Institute of medical imaging, sichuan key laboratory of medical imaging. Email:13699665433@163.com

XianHua Hu:North sichuan medical college, no. 234 fujiang road, shunqing district, 
nanchong city, sichuan province, China.Institution:Basic medical college of north sichuan medical college.Email:huxian_789@163.com

Shuai He:North sichuan medical college, no. 234 fujiang road, shunqing district, nanchong city, sichuan province, China.Institution:Department of clinical medicine, north sichuan medical college.Email:hs15583039869@163.com

Jinchuan Yu:North sichuan medical college, no. 234 fujiang road, shunqing district, nanchong city, sichuan province, China.Institution:Department of clinical medicine, north sichuan medical college.Email:411526a2364.cdb@sina.cn

Huiling Zhu:North sichuan medical college, no. 234 fujiang road, shunqing district, nanchong city, sichuan province, China.Institution:Department of clinical medicine, north sichuan medical college.Email:2043109615@qq.com

Bo $\mathrm{Mu}^{*}$ :No.55, dongshun road, gaoping district, nanchong city, sichuan province, China.Institution:Basic medical college of north sichuan medical college.Email:ppnu2013@163.com.Tel:+8613990761917.Postal Code:637000

\section{References}

1. Xi T, Zhang G. Integrated analysis of tumor differentiation genes in pancreatic adenocarcinoma [J]. Plos One, 2018, 13(3):e0193427.

2. Gemenetzis G, Groot V P, Blair A B, et al. Survival in Locally Advanced Pancreatic Cancer After Neoadjuvant Therapy and Surgical Resection.[J]. Annals of Surgery, 2019, publish ahead of print: 1 .

3. Kranenburg O. The KRAS oncogene: Past, present, and future [J]. Biochimica Et Biophysica Acta, 2005, 1756(2):81-82.

4. Muzumdar M D, Chen P Y, Dorans K J, et al. Survival of pancreatic cancer cells lacking KRAS function [J]. Nature Communications, 2017, 8(1):1090.

5. Pong W W, Jason W, Todd W, et al. F11R Is a Novel Monocyte Prognostic Biomarker 
for Malignant Glioma [J]. PLoS ONE, 2013, 8(10):e77571-.

6. Cantini L, Medico E, Fortunato S, et al. Detection of gene communities in multi-networks reveals cancer drivers [J]. Scientific Reports, 2015, 5(17386):17386.

7. Min Z, Wenting L, Bo H, et al. Overexpression of F11R in Non-Small Cell Lung Cancer Correlates with Tumor Progression [J]. PLoS ONE, 2013, 8(11):e79173-.

8. Matthaios D, Zarogoulidis P, Balgouranidou I, et al. Molecular Pathogenesis of Pancreatic Cancer and Clinical Perspectives. Oncology, 2011, 81 (3-4):259-272.

9. Saleh A G, Abdulkader A, Julien C, et al. Cten Is Targeted by Kras Signalling to Regulate Cell Motility in the Colon and Pancreas [J]. PLoS ONE, 2011, 6(6):e20919-.

10. De M G, Bardin F, Fauriat C, et al. JAM-C identifies Src family kinase-activated leukemia-initiating cells and predicts poor prognosis in acute myeloid leukemia.[J]. Cancer Research, 2017, 77(23):6627.

11. Pong W W, Jason W, Todd W, et al. F11R Is a Novel Monocyte Prognostic Biomarker for Malignant Glioma [J]. PLoS ONE, 2013, 8(10):e77571-.

12. Clasper S, Royston D, Baban D, et al. A Novel Gene Expression Profile in Lymphatics Associated with Tumor Growth and Nodal Metastasis[J]. Cancer Research, 2008, 68(18):7293-7303.

13. Norén, Elisabeth, Mellander M R, Almer S, et al. Genetic Variation and Gene Expression Levels of Tight Junction Genes Indicates Relationships Between PTEN as well as MAGI1 and Microscopic Colitis[J]. Digestive Diseases and Sciences, 2017.

14. UALCAN: A Portal for Facilitating Tumor Subgroup Gene Expression and Survival Analyses.Neoplasia. 2017 Aug;19(8):649-658.

15. Fong D , Spizzo G, Manfred Mitterer MD.Low Expression of Junctional Adhesion Molecule A Is Associated with Metastasis and Poor Survival in Pancreatic Cancer[J]. Annals of Surgical Oncology, 2012, 19(13):4330-4336.

16. John J , Sohmen R, Feuerstein J , et al. Kinetics of interaction of nucleotides with 
nucleotide-free H-ras p21[J]. Biochemistry, 1990, 29(25):6058-6065.

17. Rahib L, Smith B D , Aizenberg R , et al. Correction: Projecting Cancer Incidence and Deaths to 2030: The Unexpected Burden of Thyroid, Liver, and Pancreas Cancers in the United States[J]. Cancer Research, 2014, 74(14):4006-4006.

18. Ardito C M, Grüner BM, Takeuchi $\mathrm{K}$, et al. EGF receptor is required for KRAS-induced pancreatic tumorigenesis. [J]. Cancer Cell, 2012, 22(3):304-317.

19. Lamagna C , Hodivaladilke K M, Imhof B A , et al. Antibody against Junctional Adhesion Molecule-C Inhibits Angiogenesis and Tumor Growth[J]. Cancer Research, 2005, 65(13):5703.

20. Goetsch L, Haeuw J F , Beau-Larvor C, et al. A novel role for junctional adhesion molecule-A in tumor proliferation: Modulation by an anti-F11R monoclonal antibody[J]. International Journal of Cancer, 2013, 132(6):1463-1474.

21. Enomoto T, Fujita M, Inoue M, et al. Alterations of the p53 tumor suppressor gene and its association with activation of the c-K-ras-2 protooncogene in premalignant and malignant lesions of the human uterine endometrium[J]. Cancer Research, 1993, 53(8):1883.

22. Strohmeyer T G, Slamon D J. Proto-oncogenes and tumor suppressor genes in human urological malignancies.[J]. Journal of Urology, 1994, 151(6):1479-1497.

23. Zhang W, Flemington E K, Zhang K. Driver gene mutations based clustering of tumors: methods and applications:[J]. Bioinformatics, 2018, 34(13):i404-i411.

24. Tan C R, Yaffee P M , Jamil L H , et al. Pancreatic cancer cachexia: a review of mechanisms and therapeutics[J]. Frontiers in Physiology, 2014, 5.

25. Yuan F, Jin X L, Pathology D O, et al. Lumican protein expression in pancreatic ductal adenocarcinoma:clinical significance and correlation with expression of Ki-67,VEGF and mutated P53[J]. World Chinese Journal of Digestology, 2012, 20(12):1018.

26. Zhou Y. High-intensity focused ultrasound treatment for advanced pancreatic cancer.[J]. Gastroenterol Res Pract, 2014, 2014(5):205325. 
27. Satodahlman M, Wirth K, Yamamoto M. Role of Gene Therapy in Pancreatic CancerA Review[J]. Cancers, 2018, 10(4):103-.

28. Baine M J, Chakraborty S, Smith L M, et al. Transcriptional Profiling of Peripheral Blood Mononuclear Cells in Pancreatic Cancer Patients Identifies Novel Genes with Potential Diagnostic Utility[J]. Plos One, 2011, 6(2):e17014.

29. Molin M D, Zhang M, Wilde R F D , et al. Very Long-term Survival Following Resection for Pancreatic Cancer Is Not Explained by Commonly Mutated Genes: Results of Whole-Exome Sequencing Analysis[J]. Clinical Cancer Research, 2015, 21(8).

30. He S B, Li D C. Mutated genes in pancreatic cancer[J]. Chinese Journal of Cancer Research, 2010, 22(2):93-98.

31. Kanyama H, Tomita N, Yamano T, et al. Usefulness of Repeated Direct Intratumoral Gene Transfer Using Hemagglutinating Virus of Japan-Liposome Method for Cytosine Deaminase Suicide Gene Therapy[J]. Cancer Research, 2001, 61(1):14-18.

32. Lou E, Subramanian S, Steer C J. Pancreatic cancer: modulation of KRAS, MicroRNAs, and intercellular communication in the setting of tumor heterogeneity.[J]. Pancreas, 2013, 42(8):1218.

33. Müller M P, Jeganathan S, Heidrich A, et al. Nucleotide based covalent inhibitors of KRas can only be efficient in vivo if they bind reversibly with GTP-like affinity.[J]. Sci Rep, 2017, $7(1): 3687$.

34. Wang Y, Kaiser C E, Frett B, et al. Targeting Mutant KRAS for Anticancer Therapeutics: A Review of Novel Small Molecule Modulators[J]. Journal of Medicinal Chemistry, 2013, $56(13): 5219-5230$.

35. Ross S J, Revenko A S, Hanson L L, et al. Targeting KRAS-dependent tumors with AZD4785, a high-affinity therapeutic antisense oligonucleotide inhibitor of KRAS[J]. Science Translational Medicine, 2017, 9(394).

36. Martín-Gago P, Fansa E K, Klein C H, et al. A PDE6 $\delta$-KRas Inhibitor Chemotype with 
up to Seven H-Bonds and Picomolar Affinity that Prevents Efficient Inhibitor Release by Arl2[J]. Angewandte Chemie, 2017, 56(9):2463-2468.

37. Gupta S K, Pillarisetti K, Ohlstein E H. Platelet agonist F11 receptor is a member of the immunoglobulin superfamily and identical with junctional adhesion molecule (JAM): regulation of expression in human endothelial cells and macrophages.[J]. Iubmb Life, 2010, 50(1):51-56.

38. Lee G, Ge B, Potzold S. Widespread expressions of immunoglobulin superfamily proteins in cancer cells[J]. Cancer Immunology Immunotherapy, 2012, 61(1):89-99.

39. Samaridis J , Colonna M . Cloning of novel immunoglobulin superfamily receptors expressed on human myeloid and lymphoid cells: Structural evidence for new stimulatory and inhibitory pathways[J]. European Journal of Immunology, 2010, 27(3):660-665.

40. Zhao H, Hefen Y U, Martin T A, et al. The role of JAM-B in cancer and cancer metastasis (Review)[J]. Oncology Reports, 2016, 36(1):3-9.

41. Kedees M H, Babinska A, Swiatkowska M, et al. Expression of a recombinant protein of the platelet F11 receptor (F11R) (JAM-1/JAM-A) in insect cells: F11R is naturally phosphorylated in the extracellular domain[J]. Platelets, 2005, 16(2):99-109.

42. Miller M A, Xiao-Jiang F , Genyuan L, et al. Identifying Biological Network Structure, Predicting Network Behavior, and Classifying Network State With High Dimensional Model Representation (HDMR)[J]. PLoS ONE, 2012, 7(6):e37664-.

43. Youk J, Koh Y, Kim J W, et al. A scientific treatment approach for acute mast cell leukemia: using a strategy based on next-generation sequencing data[J]. Blood Research, 2016, 51(1):17-22.

44. Elizabeth K, Anna B, Ehrllich Y H. F11 RECEPTOR (F11R) ANTAGONISTS AS THERAPEUTIC AGENTS[J]. 2004.

45. Anna B , Ehrlich Y H , Elizabeth K. F11 receptor(f11r) antagonists as therapeutic agents:, 2007.

46. Goetsch L, Gonzalez A, Leger O, et al. A recombinant humanized anti-insulin-like 
growth factor receptor type I antibody (h7C10) enhances the antitumor activity of vinorelbine and anti-epidermal growth factor receptor therapy against human cancer xenografts.[J]. International Journal of Cancer Journal International Du Cancer, 2010, 113(2):316-28.

47. Malik - Hall M, Dina O A, Levine J D. Primary afferent nociceptor mechanisms mediating NGF - induced mechanical hyperalgesia[J]. European Journal of Neuroscience, 2015, 21(12):3387-3394. 


\section{Figures}

A

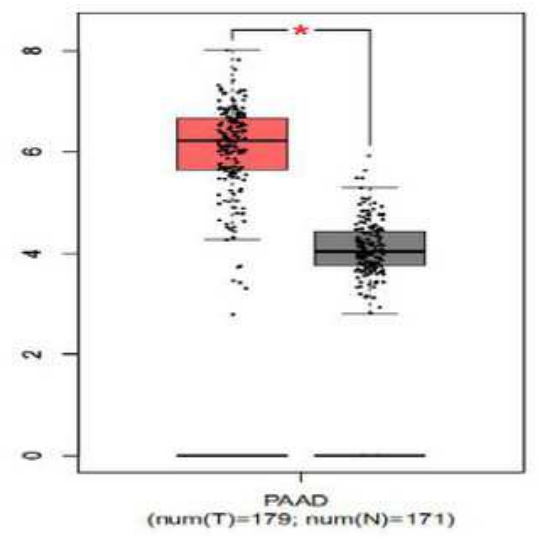

B

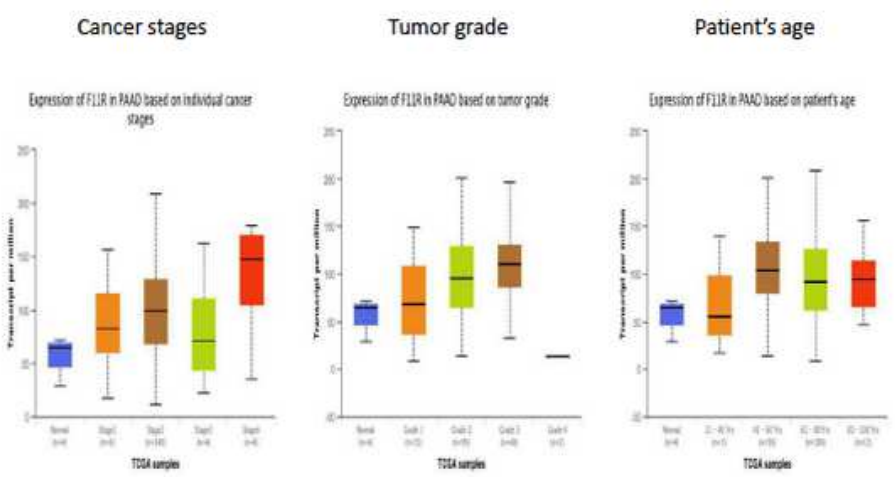

c

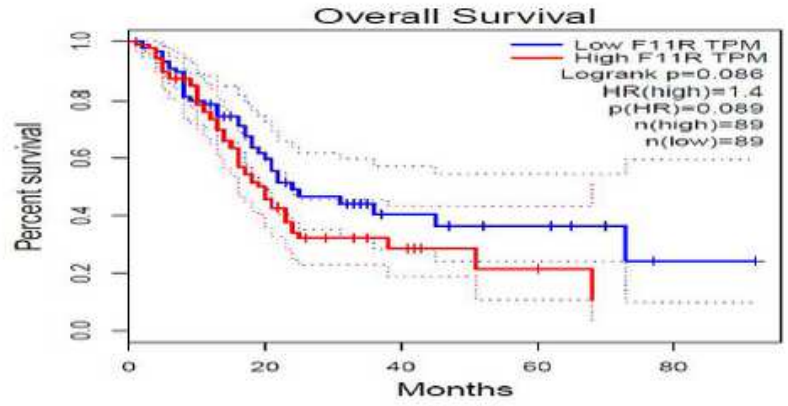

Figure 1

Bioinformatics analysis of F11R about pancreatic cancer. A: Bioinformatics analysis showed that the expression of F11R in pancreatic cancer was higher than that of normal tissue $(P<0.05)$. B: Data from the TCGA database showing the variable expression of F11R in pancreatic cancer tissue from different 
stages, different grades and different age. C:Data from the TCGA database showed that low expression of F11R prolongs the OS of pancreatic cancer patients.

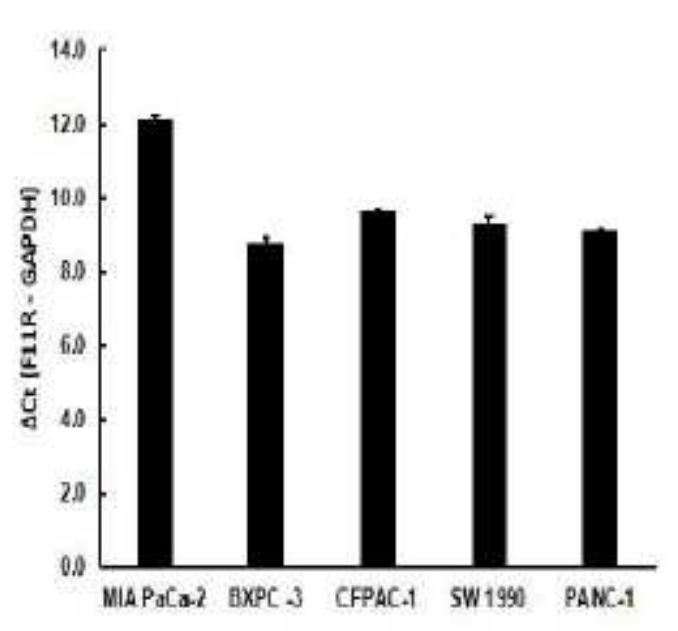

A

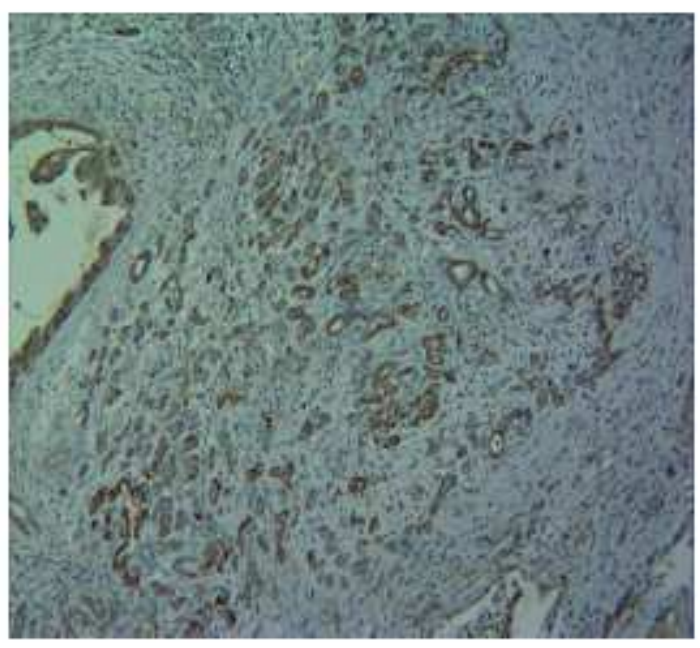

B

Figure 2

F11R expression in pancreatic cancer cell lines and PCa pathological specimens.A:q-PCR showed that F11R is highly expressed in 5(PANC-1,MIA paca-2, bxpc-3, cfpac-1, SW1990)pancreatic cancer cell lines.B: Immunohistochemical staining of F11R in pathological specimens of pancreatic cancer showed that the expression of $\mathrm{F} 11 \mathrm{R}$ was $86 \%$. 
A

RNAi-GFP White

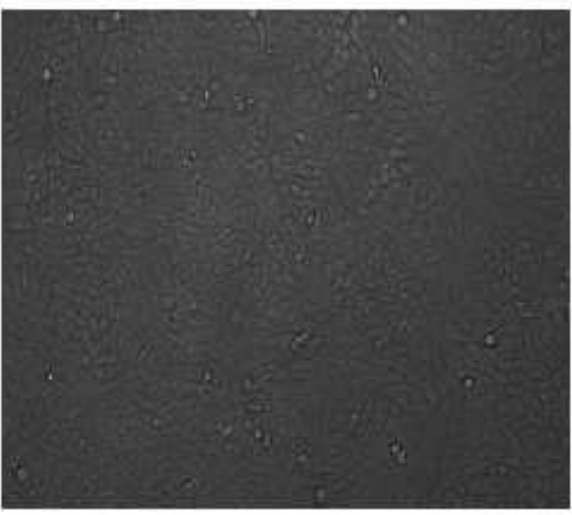

RNAi-F11R White

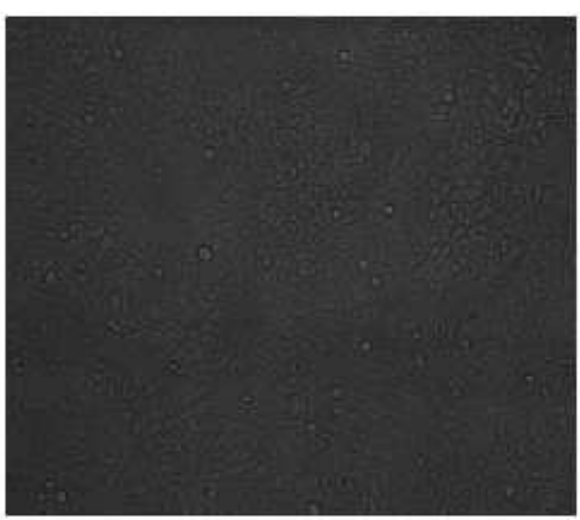

B

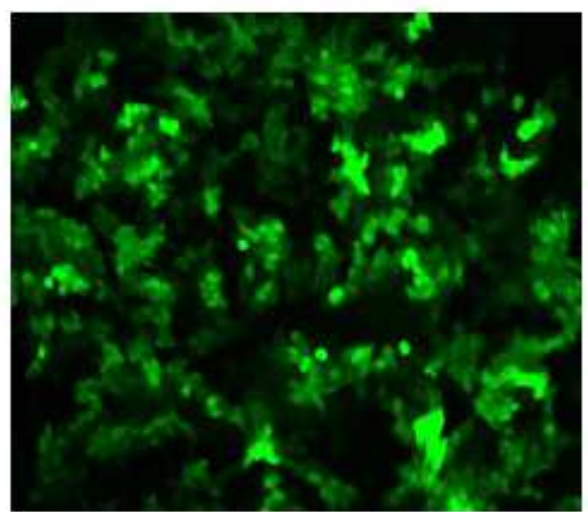

RNAi-GFP FLU

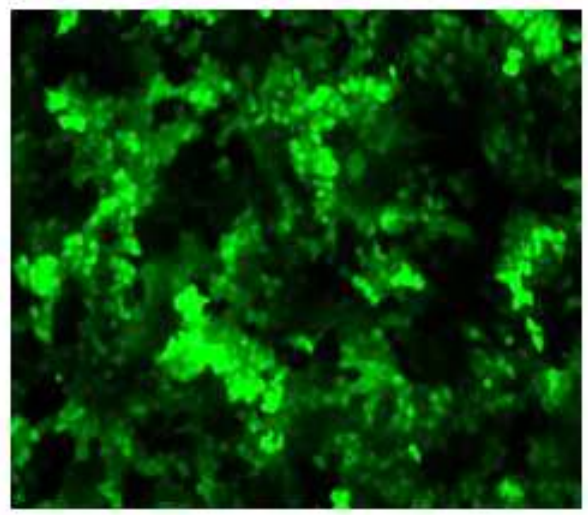

RNAi-F11R FLU

NC

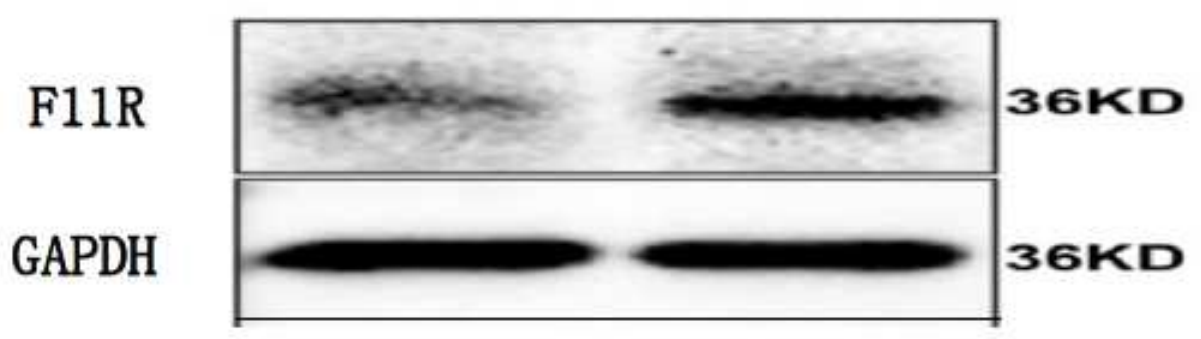

\section{Figure 3}

Knockout of PANC-1 F11R following lentivirus transfection.A: PANC-1 cells were transfected with control and F11R -/- lentiviruses for $72 \mathrm{~h}$. GFP fluorescence showed an infection efficiency $\geq 90 \%$. B: PANC-1 cells infected with the indicated lentiviruses for $72 \mathrm{~h}$ were assessed for F11R expression by western blot analysis. F11R expression in the F11R - -group was significantly reduced. 


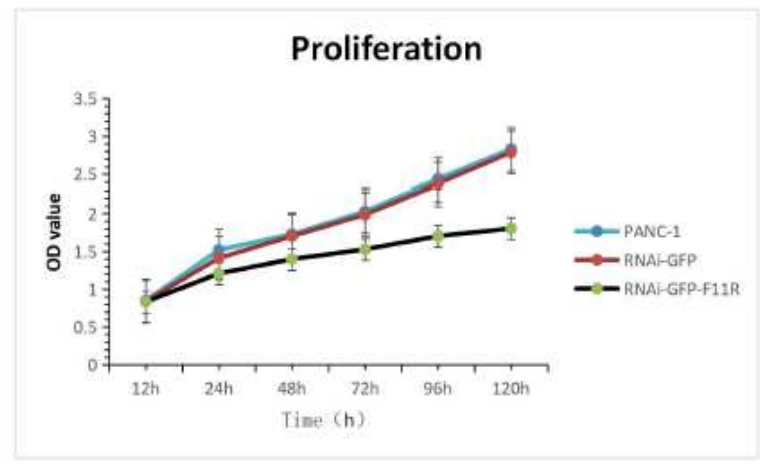

A
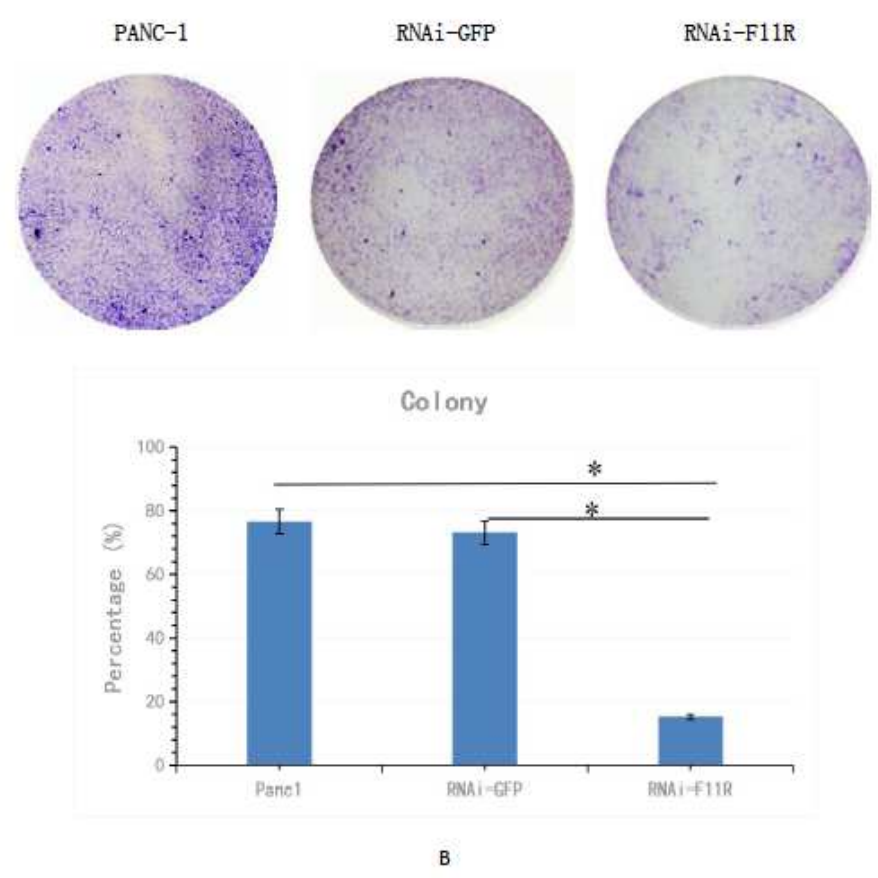

\section{Figure 4}

Cells proliferation assays. FigA:Cell proliferation in the F11R -/-group was significantly slower than the blank control and NC groups. FigB: The number of clones in the negative control group (NC) was $78.32 \%$ which decreased to $15.24 \%$ in the RNAi-GFP group. These results suggest that F11R silencing significantly inhibits the clonal formation of PANC-1 cells (* represent $P<0.05$ ). 
PANC-1
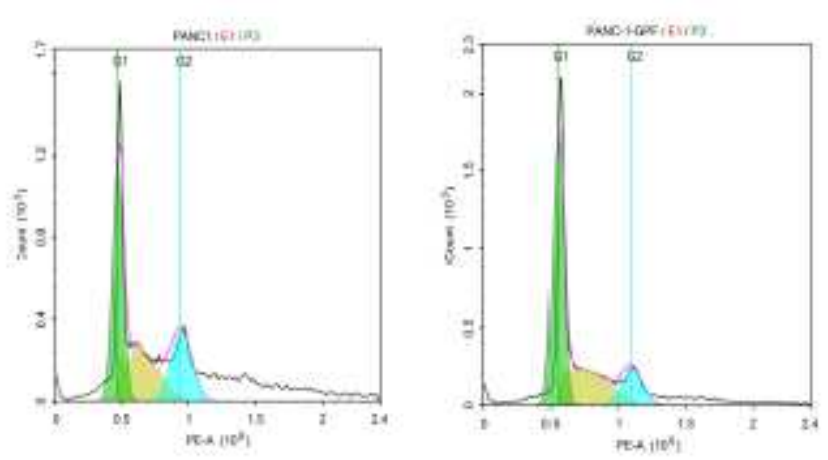

Gycle
RNAi-P11R

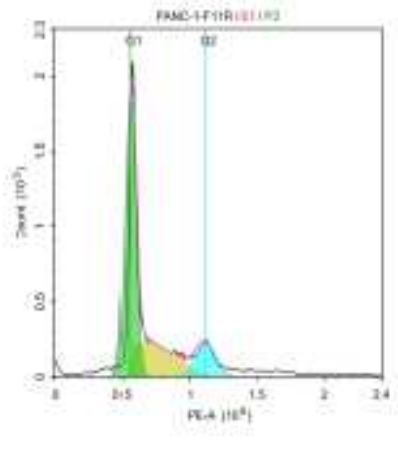

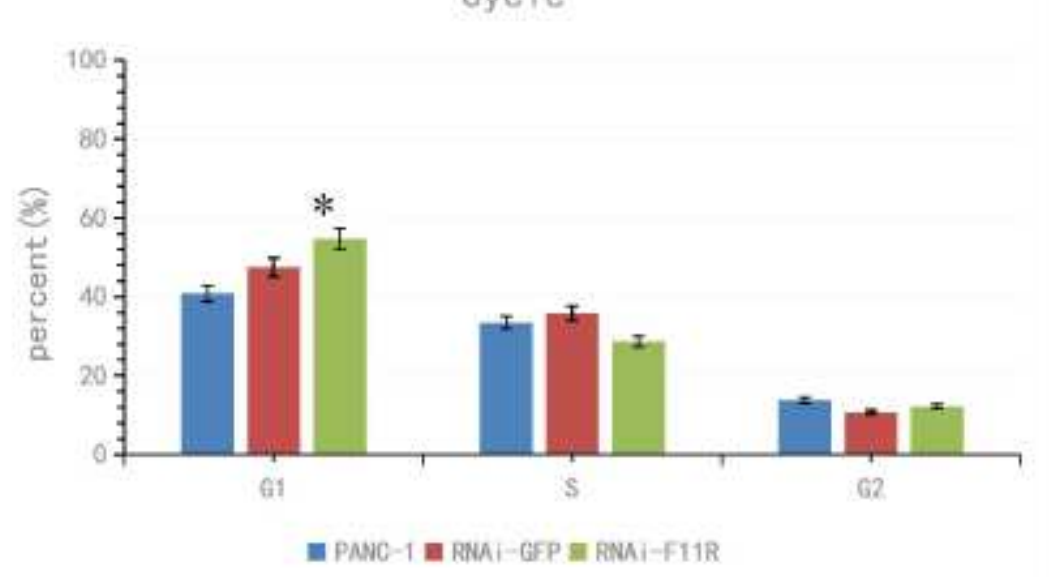

Figure 5

Flow cytometry analysis. Transfection increased the number of cells in the G0/G1 phase. Cytotoxicity was significantly higher in F11R -/- cells compared to control or NC groups. F11R silencing led to further G0/G1 phase arrest. (* represent $\mathrm{P}<0.05)$ 
PANC-1
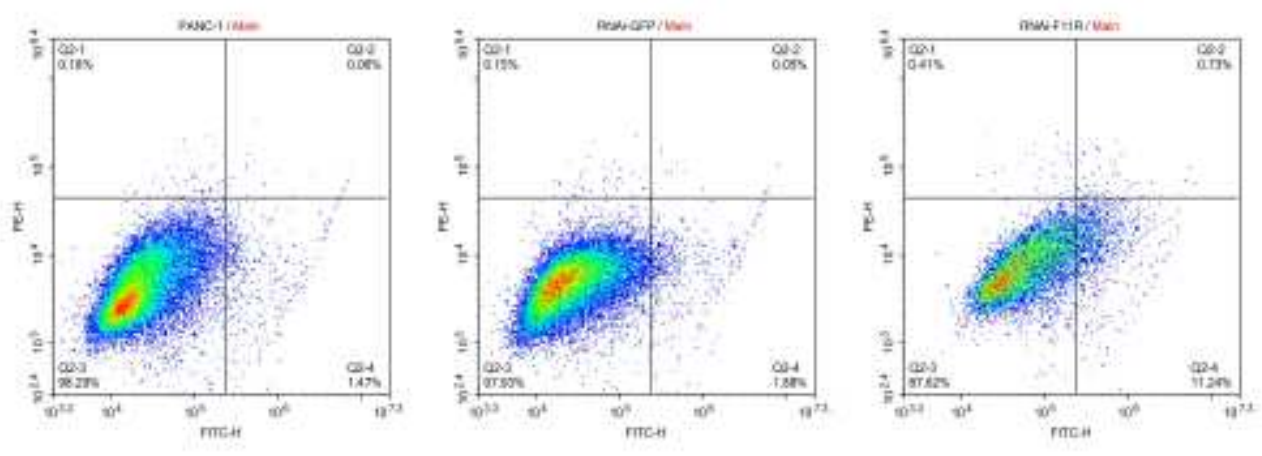

Apoptosis

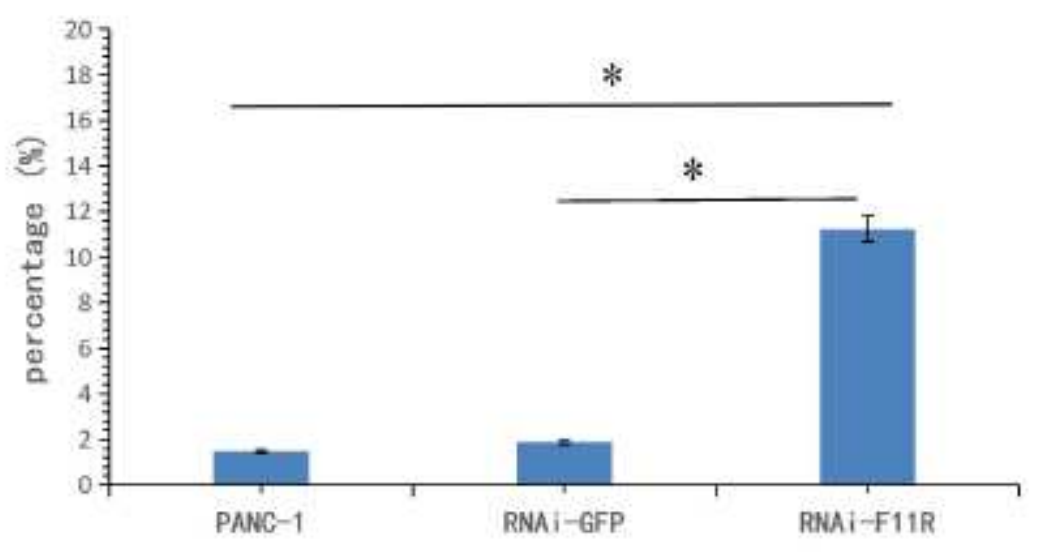

Figure 6

Apoptosis assessments. The number of apoptotic cells significantly increased following F11R silencing (lower right quadrant), as did the number of necrotic cells (upper right quadrant). Results as shown in the figure, the proportion of apoptotic cells in the untreated group was $0.74 \%$, and the proportion of apoptotic cells in the early stage of RNAi-GFP was $1.86 \%$, showing no statistical difference compared with the untreated group.The proportion of RNAi-F11R apoptotic cells in F11R -/-cells was $15.85 \%$.Compared with untreated group and NC group, the proportion of dead cells in F11R $/$ - cells increased, with statistically significant differences. $\left({ }^{*}\right.$ represent $\left.\mathrm{P}<0.05\right)$ 

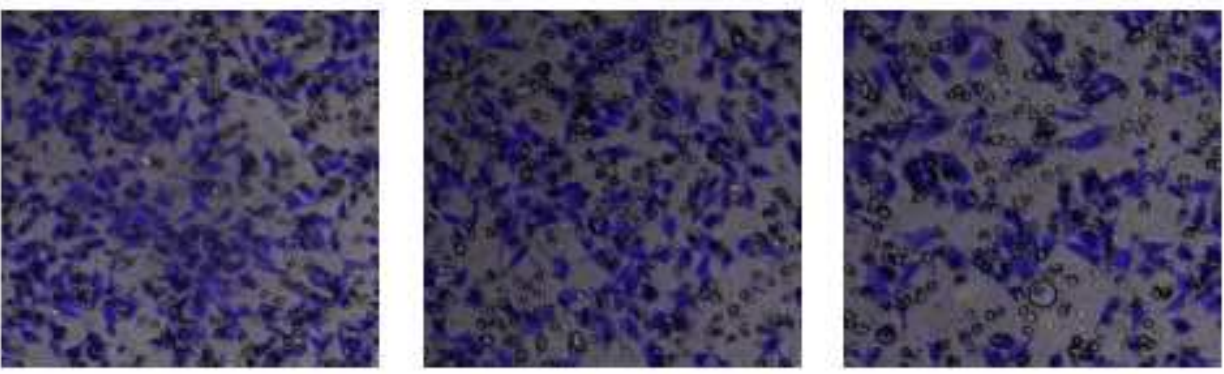

Invasion

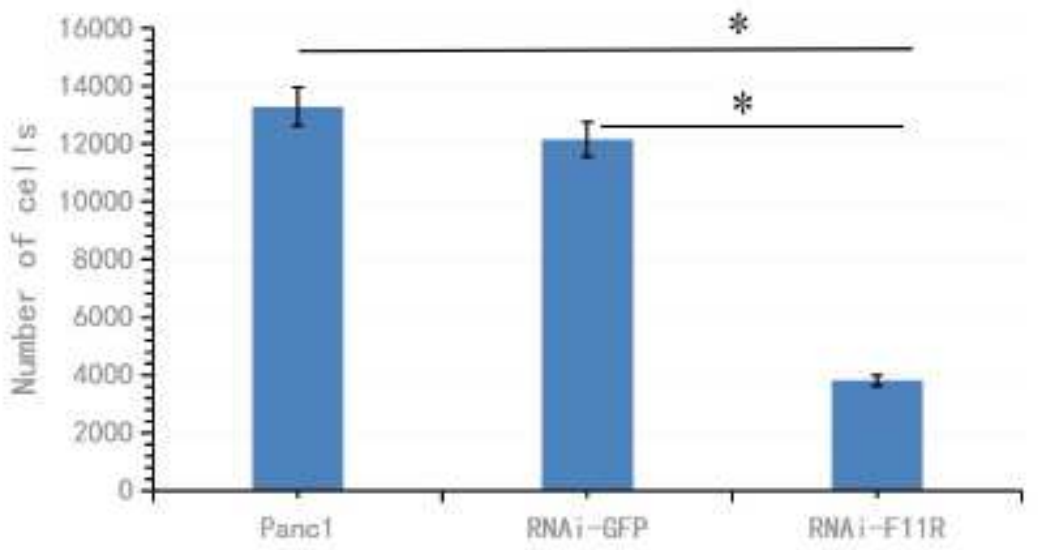

Figure 7

PANC-1 cell migration and invasion in vitro.In the F11R -/-group, the number of cells Transwell assay significantly decreased, indicating a loss of invasion. Cell numbers were calculated using ImageJ. Transwell assays showed that the number of migrating cells significantly decreased following F11R silencing. $\left({ }^{*}\right.$ represent $\left.\mathrm{P}<0.05\right)$ 

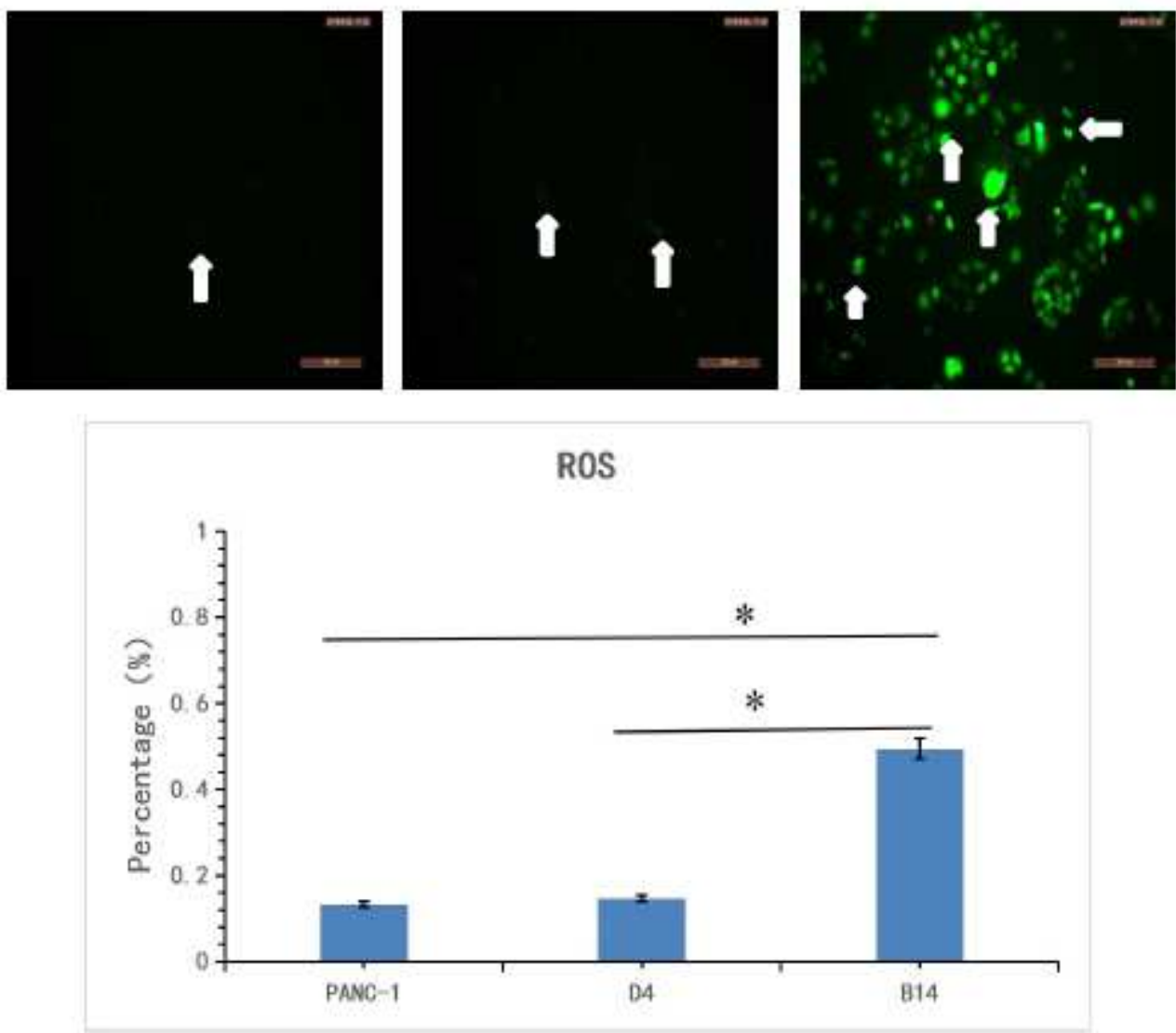

Figure 8

ROS production in PANC-1 cells. Fluorescence intensity represents intracellular ROS levels. ROS production was assessed through comparison of the fluorescent intensities of DCFH-DA staining in Control, NC and F11R groups. (* represent $\mathrm{P}<0.05)$ 